\title{
Article \\ A DNA Damage Response Gene Panel for Different Histologic Types of Epithelial Ovarian Carcinomas and Their Outcomes
}

\author{
Ying-Cheng Chiang ${ }^{1,2}{ }^{\oplus}$, Po-Han Lin ${ }^{3,4}$, Tzu-Pin Lu ${ }^{5}$, Kuan-Ting Kuo ${ }^{6}$, Yi-Jou Tai ${ }^{2,7}{ }^{\circ}$, Heng-Cheng Hsu ${ }^{7,8} \oplus^{(1)}$, \\ Chia-Ying $\mathrm{Wu}^{2,7}$, Chia-Yi Lee 7,8 (D) , Hung Shen 7,8 , Chi-An Chen ${ }^{1,2, *,+}$ and Wen-Fang Cheng ${ }^{1,2,7,9, *,+}$
}

1 Department of Obstetrics and Gynecology, College of Medicine, National Taiwan University, Taipei 100226, Taiwan; ycchiang@ntuh.gov.tw

2 Department of Obstetrics and Gynecology, National Taiwan University Hospital, Taipei 100226, Taiwan; stilabry@gmail.com (Y.-J.T.); ascheetah@msn.com (C.-Y.W.)

3 Department of Medical Genetics, National Taiwan University Hospital, Taipei 100226, Taiwan; pohanlin01@gmail.com

4 Graduate Institute of Medical Genomics and Proteomics, College of Medicine, National Taiwan University, Taipei 100025, Taiwan

5 Institute of Epidemiology and Preventive Medicine, Department of Public Health, National Taiwan University, Taipei 100025, Taiwan; tbenlu@gmail.com

6 Department of Pathology, College of Medicine, National Taiwan University, Taipei 100225, Taiwan; pathologykimo@gmail.com

7 Graduate Institute of Clinical Medicine, College of Medicine, National Taiwan University, Taipei 100225, Taiwan; b101092037@gmail.com (H.-C.H.); plzfixthecar@gmail.com (C.-Y.L.); shkt0802@gmail.com (H.S.)

check for updates

Citation: Chiang, Y.-C.; Lin, P.-H.; Lu, T.-P.; Kuo, K.-T.; Tai, Y.-J.; Hsu, H.-C.; Wu, C.-Y.; Lee, C.-Y.; Shen, H.; Chen, C.-A.; et al. A DNA Damage Response Gene Panel for Different Histologic Types of Epithelial Ovarian Carcinomas and Their Outcomes. Biomedicines 2021, 9, 1384. https://doi.org/10.3390/

biomedicines 9101384

Academic Editor: Naomi Nakayama

Received: 10 August 2021

Accepted: 29 September 2021

Published: 3 October 2021

Publisher's Note: MDPI stays neutral with regard to jurisdictional claims in published maps and institutional affiliations.

Copyright: (c) 2021 by the authors. Licensee MDPI, Basel, Switzerland. This article is an open access article distributed under the terms and conditions of the Creative Commons Attribution (CC BY) license (https:// creativecommons.org/licenses/by/ $4.0 /)$.
8 Department of Obstetrics and Gynecology, National Taiwan University Hospital, Hsin-Chu Branch, Hsin-Chu 30059, Taiwan

9 Graduate Institute of Oncology, College of Medicine, National Taiwan University, Taipei 100025, Taiwan

* Correspondence: chianchen@ntu.edu.tw (C.-A.C.); wenfangcheng@yahoo.com (W.-F.C.); Tel.: +886-2-2312-3456 (ext. 71964) (W.-F.C.)

+ Equal contribution.

Abstract: DNA damage response (DDR) is important for maintaining genomic integrity of the cell Aberrant DDR pathways lead to accumulation of DNA damage, genomic instability and malignant transformations. Gene mutations have been proven to be associated with epithelial ovarian cancer, and the majority of the literature has focused on BRCA. In this study, we investigated the somatic mutation of DNA damage response genes in epithelial ovarian cancer patients using a multiplegene panel with next-generation sequencing. In all, 69 serous, 39 endometrioid and 64 clear cell carcinoma patients were enrolled. Serous carcinoma patients $(69.6 \%)$ had higher percentages of DDR gene mutations compared with patients with endometrioid (33.3\%) and clear cell carcinoma $(26.6 \%)$ $(p<0.001$, chi-squared test). The percentages of DDR gene mutations in patients with recurrence (53.9 vs. $32.9 \% p=0.006$, chi-squared test) or cancer-related death (59.2 vs. $34.4 \% p=0.001$, chi-squared test) were higher than those without recurrence or living patients. In endometrioid carcinoma, patients with $\geq 2$ DDR gene mutations had shorter PFS ( $p=0.0035$, log-rank test) and OS ( $p=0.015$, log-rank test) than those with one mutation or none. In clear cell carcinoma, patients with $\geq 2$ DDR gene mutations had significantly shorter PFS ( $p=0.0056$, log-rank test) and OS ( $p=0.0046$, log-rank test) than those with 1 DDR mutation or none. In the EOC patients, somatic DDR gene mutations were associated with advanced-stage tumor recurrence and tumor-related death. Type I EOC patients with DDR mutations had an unfavorable prognosis, especially for clear cell carcinoma.

Keywords: epithelial ovarian cancer; DNA damage response; somatic mutation; clear cell carcinoma

\section{Introduction}

Epithelial ovarian carcinoma (EOC) is a major cause of death in women worldwide, and patients are usually diagnosed at an advanced stage with a 5-year survival of less than 50\% [1-4]. Clinical prognostic factors include cancer stage, histological subtypes, 
tumor grade, residual tumor size after debulking surgery and response to chemotherapy. Despite an initial good response to primary treatments of debulking surgery and adjuvant platinum-based chemotherapy, the majority of patients experience a cancer relapse that is resistant to salvage treatments and eventually die of the disease [4,5].

Precision medicine is the current direction for cancer management depending on the specific genetic or molecular features of cancer. There are several subtypes of EOChigh-grade serous, clear cell, endometrioid, mucinous and low-grade serous-that could be viewed as distinct diseases for their differences in clinical course and pathological features [6,7]. To date, the most promising target therapies for EOC are anti-angiogenesis agents and poly ADP-ribose polymerase inhibitors (PARPi). Bevacizumab in combination with chemotherapy has demonstrated improved progression-free survival, and an overall survival benefit in high-risk patients [8-10]. Maintenance therapy with PARPi has revised the management of EOC in newly diagnosed and recurrent diseases. The identification of $B R C A$ mutations or homologous recombination deficiency (HRD) status is critical for selecting potential patients, but both positive and negative patients as defined by current HRD assays benefited from PARPi [11-15].

DNA damage response (DDR) is important for maintaining a cell's genomic integrity, and the DDR pathway is composed of various molecules that detect DNA damage, activate cell-cycle checkpoints, trigger apoptosis, and coordinate DNA repair [16-18]. Several exogenous or endogenous sources (e.g., oxidative damage, radiation, ultraviolet light, cytotoxic materials, replication errors) may result in DNA damage that may eventually lead to genomic instability and cell death [19]. DDR consists of several pathways, including base excision (BER), mismatch (MMR) and nucleotide excision repair (NER); translesion synthesis (TLS) for single-strand break repair; homologous recombination (HR) and nonhomologous DNA end joining (NHEJ) for double-strand break repair; and cell cycle regulation (CCR) $(27,28)$. Homologous recombination is an error-proof repair pathway to restore the original sequence at the double-strand DNA break. BRCA 1/2 genes participating in HR and maintaining PARPi therapy for $B R C A$-mutated EOC is a good example of synthetic lethality [20]. Several other DDR genes have been identified as potential targets for novel cancer therapy under clinical investigation [16,17]. Understanding the complex DDR pathways is helpful for exploring the feasibility of novel DDR inhibitors in clinical practice. In the study, we investigated the somatic mutations of DDR genes in 172 EOC patients using a targeted DDR gene panel using a next-generation sequencing method. The correlation of the somatic DDR gene mutations, clinical parameters and outcomes was analysed.

\section{Materials and Methods}

\subsection{Patients and Specimens}

The study protocol was approved by the National Taiwan University Hospital Research Ethics Committee (201509042RINA, approved on 24 November 2015 and 201608025RINA, approved on 07 October 2016). Informed consent from all participants was obtained and the methods were performed in accordance with the guidelines and regulations. From December 2015 to October 2018, 172 women diagnosed with epithelial ovarian cancer who had received debulking surgery and adjuvant chemotherapy were enrolled. The cancerous tissue specimens collected during debulking surgery were immediately frozen in liquid nitrogen and stored at $-70^{\circ} \mathrm{C}$. A portion of the tissue specimens were sent for pathological examinations to confirm the diagnosis and ensure tumorous tissue sufficient for the following experiments. Clinical data were obtained from medical records, including age, cancer stage, the findings during debulking surgery, treatment course and recurrence. Optimal debulking surgery was defined as a maximal residual tumor size $<1 \mathrm{~cm}$ following surgery. The tumor grade based on International Union Against Cancer criteria, and cancer stage was based on International Federation of Gynecology and Obstetrics (FIGO) criteria [21]. All patients received platinum-based adjuvant chemotherapy and regular follow-ups after primary treatments. Recurrence was defined as abnormal results from imaging studies (including computerized tomography or magnetic resonance imaging), 
elevated CA-125 (more than twice the upper normal limit) for two consecutive tests in 2week intervals, or a biopsy-proven disease. Progression-free survival (PFS) was defined as the time from the date of primary treatment completion to the date of confirmed recurrence, disease progression or last follow-up. Overall survival (OS) was defined as the period from surgery to the date of death related to EOC or the date of last follow-up.

\subsection{The Panel of DNA Damage Repair Genes}

We selected 60 genes involved in DNA damage response (DDR) for the gene panel (Table 1), including genes of homologous recombination (HR), nonhomologous DNA end joining (NHEJ), base excision repair (BER), mismatch repair (MMR), nucleotide excision repair (NER), translesion synthesis (TLS) and cell cycle regulation (CCR) [16,17].

Table 1. List of the DNA damage response (DDR) gene panel.

\begin{tabular}{|c|c|c|c|}
\hline Gene & DDR Pathway & Gene & DDR Pathway \\
\hline ATM & CCR & ku70/XRCC6 & NHEJ \\
\hline BARD1 & HR & ku80/XRCC5 & NHEJ \\
\hline$B R C A 1$ & HR & MDM4 & CCR \\
\hline BRCA2/FANCD1 & HR & MLH1 & MMR \\
\hline BRIP1/FANCJ & HR & MLH3 & MMR \\
\hline CHEK2 & CCR & MRE11 & HR \\
\hline$D D B 1$ & NER & $\mathrm{MSH} 2$ & MMR \\
\hline$D D B 2$ & NER & MSH3 & MMR \\
\hline ERCC1 & NER & MSH6 & MMR \\
\hline ERCC2/XPD & NER & MUTYH & BER \\
\hline$E R C C 3 / X P B$ & NER & $N B N$ & HR \\
\hline ERCC4 & NER & NBS1 & HR \\
\hline ERCC5/BIVM & NER & OGG1 & BER \\
\hline ERCC6/CSB & NER & PMS1 & MMR \\
\hline ERCC8/CSA & NER & PMS2 & MMR \\
\hline FANCA & $\mathrm{HR}$ & POLD1 & TLS \\
\hline FANCB & HR & POLE & TLS \\
\hline FANCC & HR & POLB & TLS \\
\hline FANCD1/BRCA2 & HR & POLH & TLS \\
\hline FANCD2 & HR & POLK & TLS \\
\hline FANCE & HR & RAD50 & HR \\
\hline FANCF & HR & RAD51 & HR \\
\hline FANCG/XRCC & HR & RAD51C/FANCO & HR \\
\hline FANCI & HR & RAD51D & $\mathrm{HR}$ \\
\hline FANCJ/BRIP1 & HR & TP53 & CCR \\
\hline FANCL/PHF9 & HR & $X P A$ & NER \\
\hline FANCM & HR & $X P C$ & NER \\
\hline FANCN/PALB2 & HR & XRCC2 & NHEJ \\
\hline FANCO/RAD51C & HR & XRCC3 & NHEJ \\
\hline FANCP/SLX4 & HR & XRCC4 & NHEJ \\
\hline
\end{tabular}

Note: BER: base excision repair; CCR: cell cycle regulation; DDR: DNA damage repair; HR: homologous recombination; MMR: mismatch repair; NER: nucleotide excision repair; NHEJ: nonhomologous DNA end joining; TLS: translesion synthesis.

\subsection{Genomic DNA Extraction}

Genomic DNA was isolated using a QIAGEN Genomic DNA extraction kit according to the manufacturer's instructions (Qiagen Inc., Valencia, CA, US). The purity and concentration of the genomic DNA were checked by agarose gel electrophoresis and the $\mathrm{OD}_{260 / 280}$ ratio.

\subsection{Library Preparation, Next-Generation Sequencing, and Sequence Mapping}

The genomic DNA was fragmented with Covaris fragmentation protocol (Covaris, Inc., Woburn, MA, US). The size of the fragmented genomic DNA was checked by Agilent Bioanalyzer 2100 (Agilent Technologies, Inc., Santa Clara, CA, US) and NanoDrop 
spectrophotometer (Thermo Fisher Scientific, Inc., Wilmington, DE, US). The target gene library was generated with NimblGen capture kits (Roche NimblGen, Inc. Hacienda Dr Pleasanton, CA, US). The samples were sequenced by Illumina MiSeq with paired-end reads of 300 nucleotides.

The analysis algorithm was conducted according to our previous protocol [22]. Briefly, the raw sequencing data were aligned with the reference human genome (Feb. 2009, GRCh37/hg19) with Burrows-Wheeler Aligner software (version 0.5.9) [23]. SAM tools (version 0.1.18) was used for data conversion, sorting, and indexing [24]. For single nucleotide polymorphisms (SNPs) and small insertion/deletions (indels), Genome Analysis Toolkit (GATK; version 2.7) was used for variant calling with Base/indel-calibrator and HaplotypeCaller. Pindel or Breakdancer software were used for structural variants larger than 100 bp which cannot be identified by GATK, such as large deletions, insertions and duplications [25]. After variant calling, ANNOVAR was used for annotation of the genetic variants [26,27]. The dbSNP, Exome sequencing Project 6500 (ESP6500) and the 1000 Genomes variant dataset were used to filter common variants of sequencing results.

\subsection{Variant Classification}

The sequence variants were classified according to the IARC variant classification [28]. The pathogenic mutations were defined as large-scale deletion, frame-shift mutation, nonsense mutation, genetic variants associated with uncorrected splicing and mutations affecting protein function demonstrated by functional analyses. The pathogenic and likely pathogenic mutations were used as deleterious mutations in our study. An allele frequency greater than 0.01 in the general population in the 1000 Genomes variant dataset or ESP6500 database were considered benign or likely benign genetic variants. Silent and intronic variants that did not affect splicing were also considered benign or likely benign. Other variants, mainly missense mutations without known functional data, were considered as variants of uncertain significance (VUSs). To reduce their number, bioinformatics analyses, including PolyPhen2 and SIFT, were used to evaluate potential pathogenicity [29-31]. The VUSs were suspected of being deleterious mutations if they met two criteria: (1) a population frequency of less than 0.01 in the 1000 Genomes and ESP6500 databases and (2) a bioinformatics analysis result with a SIFT score less than 0.05 and a polyphen 2 score greater than 0.95 .

\subsection{Statistical Analysis}

All statistical analyses were performed using the Statistical Package for Social Sciences software package (IBM SPSS Statistics for Windows, Version 22.0. IBM Corp. Armonk, NY, US) and R (version 3.1.2, The R Foundation for Statistical Computing, Institute for Statistics and Mathematics, Wirtschaftsuniversität Wien, Welthandelsplatz Vienna, Austria). One-way ANOVA was used to compare continuous variables and a chi-squared test was used for categorical variables. Survival curves were generated using the Kaplan-Meier method, and differences were calculated using the log-rank test. A multivariate Cox's regression model was used to evaluate the prognostic factors for progression-free survival (PFS) and overall survival (OS). Statistical significance was set as a $p$ value of less than 0.05 .

\section{Results}

\subsection{Clinical Characteristics of the Patients}

There were 172 EOC patients enrolled: 69 serous, 39 endometrioid and 64 clear cell carcinomas (Table 2). There were 68 high-grade serous carcinomas (type II tumor) and 104 type I tumors. The median age was 52, and the median pre-treatment CA125 value was $400 \mathrm{U} / \mathrm{mL} ; 59.9 \%$ were diagnosed at an advanced cancer stage, and $65.1 \%$ had undergone optimal debulking surgery; $59.3 \%$ had disease recurrence, and $44.2 \%$ died of EOC. All patients received adjuvant platinum and paclitaxel chemotherapy. 
Table 2. Characteristics of the epithelial ovarian cancer patients.

\begin{tabular}{cc}
\hline Patient Numbers & $\mathbf{1 7 2}$ \\
\hline Median Age (years old) & $52(29-85)$ \\
Median CA 125 (U/mL) & $400(12-7265)$ \\
Histology & $69(40.1 \%)$ \\
Serous carcinoma & $39(22.7 \%)$ \\
Endometrioid carcinoma & $64(37.2 \%)$ \\
Clear cell carcinoma & \\
FIGO stage & $69(40.1 \%)$ \\
Early & $103(59.9 \%)$ \\
Advanced & \\
Grade & $29(16.9 \%)$ \\
Low & $143(83.1 \%)$ \\
High & $112(65.1 \%)$ \\
Debulking surgery & $60(34.9 \%)$ \\
Optimal & \\
Suboptimal & $102(59.3 \%)$ \\
Recurrence & $70(40.7 \%)$ \\
Yes & \\
No & $76(44.2 \%)$ \\
Death & $96(55.8 \%)$ \\
Yes &
\end{tabular}

\subsection{Deleterious DDR Gene Mutations}

As shown in Table 3, 114 deleterious somatic mutations were identified from 26 genes of our 60-gene DDR panel in 78 EOC patients: 27 nonsense mutations in 23 patients, 28 frameshift mutations in 20,28 missense mutations in 26 patients and 31 mutations involving uncorrected splicing in 29 patients. There were single-gene mutations in 57 patients, and multiple-gene mutations in 21: 2 mutations in 14 patients, 3 mutations in 2, 4 mutations in 3,5 mutations in 1 and 6 mutations in 1 patient (Figure 1). We also identified 109 missense mutations classified as variants of uncertain significance (VUSs) with the potential of being deleterious mutations after searching the database (http:/ / www.ncbi.nlm.nih.gov/snp, accessed on 28 September 2021) and bioinformatic analyses (Table S1 and Figure S1).

The pattern of prevalent mutated DDR genes was different among the histological subtypes (Figure 1). The proportion of wild type DDR genes was $54.7 \%$ in all EOC patients; $30.4 \%$ in serous carcinoma, $66.7 \%$ in endometrioid carcinoma and $73.4 \%$ in clear cell carcinoma. The top three prevalent mutated DDR genes were TP53 (27.9\%), MUTYH (6.4\%) and BRCA2 (5.8\%) for all patients. Serous carcinoma-TP53 (56.5\%), BRCA2 (5.8\%) and RAD51C (5.8\%); endometrioid carcinoma-TP53 (15.4\%), ATM (12.8\%) and MSH2 (7.7\%); clear cell carcinoma-MUTYH (9.4\%), TP53 (4.7\%), BRCA2 (3.1\%) and ERCC8 (3.1\%). The top three prevalent mutated subgroups of DDR genes were CCR $(30.8 \%), \mathrm{HR}(10.5 \%)$ and BER $(7.0 \%)$ for all patients. Serous carcinoma-CCR $(58.05 \%)$, HR $(15.9 \%)$ and BER (5.8\%); endometrioid carcinoma-CCR (23.1\%), MMR (15.4\%) and HR (7.7\%); clear cell carcinoma-BER $(9.4 \%)$, CCR $(6.3 \%)$ and HR $(6.3 \%)$. For detailed information, please refer to Table S2 and Figure S2. 
Table 3. The deleterious DDR gene mutations in the patients.

\begin{tabular}{|c|c|c|c|c|c|}
\hline Gene & Mutation & Transcript & gDNA/cDNA & Amino Acid & Reported/Novel \\
\hline ATM & frameshift deletion & NM_000051 & c.1402_1403del & p.K468fs & rs587781347 \\
\hline ATM & frameshift deletion & NM_000051 & c.8426delA & p.Q2809fs & rs587782558 \\
\hline ATM & frameshift insertion & NM_000051 & c.4736dupA & p.Q1579fs & rs864622164 \\
\hline ATM & missense mutation & NM_000051 & c.C6200A & p.A2067D & rs397514577 \\
\hline ATM & nonsense mutation & NM_000051 & c.C5188T & p.R1730X & rs764389018 \\
\hline ATM & nonsense mutation & NM_000051 & c.C850T & p.Q284X & rs757782702 \\
\hline$B A R D 1$ & frameshift insertion & NM_000465 & c.70_71insGT & p.P24fs & NA \\
\hline BRCA1 & nonsense mutation & NM_007294 & c.3531dupT & p.S1178_K1179delinsX & NA \\
\hline$B R C A 1$ & nonsense mutation & NM_007294 & c.G2635T & p.E879X & rs80357251 \\
\hline BRCA2 & frameshift deletion & NM_000059 & c.1585delT & p.F529fs & NA \\
\hline$B R C A 2$ & frameshift insertion & NM_000059 & c.7407dupT & p.T2469fs & rs397507916 \\
\hline$B R C A 2$ & nonsense mutation & NM_000059 & c. 4965 delC & p.Y1655X & rs80359475 \\
\hline$B R C A 2$ & nonsense mutation & NM_000059 & c.A5623T & p.K1875X & NA \\
\hline$B R C A 2$ & nonsense mutation & NM_000059 & c.C2590T & p.Q864X & rs1060502414 \\
\hline BRCA2 & nonsense mutation & NM_000059 & c.C6952T & p.R2318X & rs80358920 \\
\hline$B R C A 2$ & nonsense mutation & NM_000059 & c.G3922T & p.E1308X & rs 80358638 \\
\hline BRIP1 & frameshift insertion & NM_032043 & c.394dupA & p.T132fs & rs587781416 \\
\hline CHEK2 & splicing & NM_007194 & g. 29130716 C>G & & NA \\
\hline ERCC8 & frameshift deletion & NM_000082 & c.191_195del & p.S64fs & NA \\
\hline ERCC8 & splicing & NM_000082 & c. $1123-2->T$ & & NA \\
\hline ERCC8 & splicing & NM_000082 & c. $1123-2->\mathrm{T}$ & & NA \\
\hline ERCC8 & splicing & NM_000082 & c. $1123-2->\mathrm{T}$ & & NA \\
\hline ERCC8 & splicing & NM_000082 & c. $1123-2->\mathrm{T}$ & & rs777444521 \\
\hline FANCC & nonsense mutation & NM_000136 & c.G1225T & p.E409X & NA \\
\hline FANCG & splicing & NM_004629 & c. $511-2->C$ & & NA \\
\hline FANCI & splicing & NM_001113378 & c. $3187-2 A>G$ & & NA \\
\hline FANCM & frameshift deletion & NM_020937 & c.3998delA & p.Q1333fs & rs746983128 \\
\hline MLH1 & frameshift deletion & NM_000249 & c.1771delG & p.D591fs & NA \\
\hline MLH1 & splicing & NM_000249 & c. $2104-2 A>G$ & & rs267607889 \\
\hline MLH1 & splicing & NM_000249 & c. $790+2 \mathrm{~T}>\mathrm{C}$ & & rs267607790 \\
\hline MLH3 & missense mutation & NM_001040108 & c.G2221T & p.V741F & rs28756990 \\
\hline$M L H 3$ & missense mutation & NM_001040108 & c.G2221T & p.V741F & rs28756990 \\
\hline MLH3 & missense mutation & NM_001040108 & c.G2221T & p.V741F & rs28756990 \\
\hline MLH3 & missense mutation & NM_001040108 & c.G2221T & p.V741F & rs 28756990 \\
\hline MRE11 & frameshift insertion & NM_005590 & c.1222dupA & p.T408fs & rs774440500 \\
\hline MSH2 & nonsense mutation & NM_000251 & c.C226T & p.Q76X & rs63750042 \\
\hline MSH2 & nonsense mutation & NM_000251 & c.G1738T & p.E580X & rs63751411 \\
\hline $\mathrm{MSH} 2$ & splicing & NM_000251 & c. $943-1 G>C$ & & rs12476364 \\
\hline MSH3 & frameshift deletion & NM_002439 & c.1141delA & p.K381fs & rs587776701 \\
\hline MSH6 & frameshift insertion & NM_001281492 & c.2916dupT & p.T972fs & NA \\
\hline MSH6 & nonsense mutation & NM_001281492 & c.G726A & p.W242X & NA \\
\hline MSH6 & splicing & NM_001281492 & 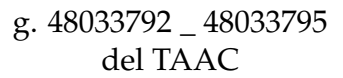 & & NA \\
\hline MUTYH & missense mutation & NM_001128425 & c.G1187A & p.G396D & rs36053993 \\
\hline MUTYH & nonsense mutation & NM_001128425 & c.G467A & p.W156X & rs762307622 \\
\hline MUTYH & splicing & NM_001128425 & c. $576+1 G>C$ & & NA \\
\hline MUTYH & splicing & NM_001128425 & c. $934-2 A>G$ & & rs77542170 \\
\hline MUTYH & splicing & NM_001128425 & c. $934-2 A>G$ & & rs77542170 \\
\hline MUTYH & splicing & NM_001128425 & c. $934-2 A>G$ & & rs77542170 \\
\hline MUTYH & splicing & NM_001128425 & c. $934-2 A>G$ & & rs77542170 \\
\hline MUTYH & splicing & NM_001128425 & c. $934-2 A>G$ & & rs77542170 \\
\hline MUTYH & splicing & NM_001128425 & c. $934-2 A>G$ & & rs77542170 \\
\hline MUTYH & splicing & NM_001128425 & c. $934-2 A>G$ & & rs77542170 \\
\hline MUTYH & splicing & NM_001128425 & c. $934-2 A>G$ & & rs 77542170 \\
\hline OGG1 & nonsense mutation & NM_016819 & c.A974G & p.X325W & NA \\
\hline POLD1 & splicing & NM_002691 & c. $2954-1 G>-$ & & NA \\
\hline RAD50 & frameshift deletion & NM_005732 & c. $2157 \mathrm{delA}$ & p.L719fs & NA \\
\hline RAD50 & frameshift deletion & NM_005732 & c.536delT & p.I179fs & NA \\
\hline
\end{tabular}


Table 3. Cont.

\begin{tabular}{|c|c|c|c|c|c|}
\hline Gene & Mutation & Transcript & gDNA/cDNA & Amino Acid & Reported/Novel \\
\hline RAD50 & frameshift insertion & NM_005732 & exon13:c.2157dupA & p.L719fs & rs397507178 \\
\hline RAD51C & frameshift insertion & NM_058216 & c.390dupA & p.G130fs & rs730881940 \\
\hline RAD51C & nonsense mutation & NM_058216 & c. $\mathrm{T} 833 \mathrm{G}$ & p.L278X & NA \\
\hline RAD51C & splicing & NM_058216 & c. $905-2 \mathrm{~A}>\mathrm{C}$ & & NA \\
\hline RAD51C & splicing & NM_058216 & c. $905-2 \mathrm{~A}>\mathrm{C}$ & & NA \\
\hline RAD51D & splicing & NM_002878 & c. $480+1 G>A$ & & NA \\
\hline TP53 & frameshift deletion & NM_000546 & c.102delC & p.P34fs & NA \\
\hline TP53 & frameshift deletion & NM_000546 & c.121delG & p.D41fs & NA \\
\hline TP53 & frameshift deletion & NM_000546 & c. 216 delC & p.P72fs & NA \\
\hline TP53 & frameshift deletion & NM_000546 & c.257_272del & p.A86fs & NA \\
\hline TP53 & frameshift deletion & NM_000546 & c.501delG & p.Q167fs & NA \\
\hline TP53 & frameshift deletion & NM_000546 & c.539_549del & p.E180fs & NA \\
\hline TP53 & frameshift insertion & NM_000546 & c.102-dupC & p.L35fs & NA \\
\hline TP53 & frameshift insertion & NM_000546 & c.455dupC & p.P152fs & NA \\
\hline TP53 & frameshift insertion & NM_000546 & c.498dupA & p.Q167fs & NA \\
\hline TP53 & frameshift insertion & NM_000546 & c.889dupC & p.H297fs & NA \\
\hline TP53 & missense mutation & NM_000546 & c.A488G & p.Y163C & rs148924904 \\
\hline TP53 & missense mutation & NM_000546 & c.A578G & p.H193R & rs786201838 \\
\hline TP53 & missense mutation & NM_000546 & c.A659C & p.Y220S & rs121912666 \\
\hline TP53 & missense mutation & NM_000546 & c.A659G & p.Y220C & rs121912666 \\
\hline TP53 & missense mutation & NM_000546 & c.A659G & p.Y220C & rs121912666 \\
\hline TP53 & missense mutation & NM_000546 & c.A736G & p.M246V & rs483352695 \\
\hline TP53 & missense mutation & NM_000546 & c.A838G & p.R280G & rs753660142 \\
\hline TP53 & missense mutation & NM_000546 & c.C380T & p.S127F & rs730881999 \\
\hline TP53 & missense mutation & NM_000546 & c.C $451 \mathrm{~T}$ & p.P151S & rs28934874 \\
\hline TP53 & missense mutation & NM_000546 & c. $\mathrm{C} 844 \mathrm{~T}$ & p.R282W & rs28934574 \\
\hline TP53 & missense mutation & NM_000546 & c.C $844 \mathrm{~T}$ & p.R282W & rs 28934574 \\
\hline TP53 & missense mutation & NM_000546 & c.G412C & p.A138P & rs28934875 \\
\hline TP53 & missense mutation & NM_000546 & c.G524A & p.R175H & rs 28934578 \\
\hline TP53 & missense mutation & NM_000546 & c.G524A & p.R175H & rs28934578 \\
\hline TP53 & missense mutation & NM_000546 & c.G638T & p.R213L & rs587778720 \\
\hline TP53 & missense mutation & NM_000546 & c.G730A & p.G244S & rs1057519989 \\
\hline TP53 & missense mutation & NM_000546 & c.G743A & p.R248Q & rs11540652 \\
\hline TP53 & missense mutation & NM_000546 & c.G743A & p.R248Q & rs11540652 \\
\hline TP53 & missense mutation & NM_000546 & c.G818A & p.R273H & rs 28934576 \\
\hline TP53 & missense mutation & NM_000546 & c.G818A & p.R273H & rs 28934576 \\
\hline TP53 & missense mutation & NM_000546 & c.G836A & p.G279E & rs1064793881 \\
\hline TP53 & missense mutation & NM_000546 & c.G856A & p.E286K & rs786201059 \\
\hline TP53 & nonsense mutation & NM_000546 & c.588_589insTGA & p.V197delinsX & NA \\
\hline TP53 & nonsense mutation & NM_000546 & c. 912 dupT & p.K305_R306delinsX & NA \\
\hline TP53 & nonsense mutation & NM_000546 & c.C430T & p.Q144X & NA \\
\hline TP53 & nonsense mutation & NM_000546 & c.C499T & p.Q167X & NA \\
\hline TP53 & nonsense mutation & NM_000546 & c.C574T & p.Q192X & NA \\
\hline TP53 & nonsense mutation & NM_000546 & c.C586T & p.R196X & rs397516435 \\
\hline TP53 & nonsense mutation & NM_000546 & c.C637T & p.R213X & rs397516436 \\
\hline TP53 & nonsense mutation & NM_000546 & c.G272A & p.W91X & NA \\
\hline TP53 & nonsense mutation & NM_000546 & c.G438A & p.W146X & NA \\
\hline TP53 & nonsense mutation & NM_000546 & c.G859T & p.E287X & NA \\
\hline TP53 & nonsense mutation & NM_000546 & c.G880T & p.E294X & rs1057520607 \\
\hline TP53 & splicing & NM_000546 & c. $376-1 G>T$ & & NA \\
\hline TP53 & splicing & NM_000546 & c. $672+1 G>A$ & & rs863224499 \\
\hline TP53 & splicing & NM_000546 & c. $993+2 \mathrm{~T}>\mathrm{G}$ & & NA \\
\hline TP53 & splicing & NM_000546 & c. $993+2 \mathrm{~T}>\mathrm{G}$ & & NA \\
\hline TP53 & splicing & NM_000546 & c. $993+1 G>T$ & & rs11575997 \\
\hline ТР53 & splicing & NM_000546 & $\begin{array}{c}\text { g.75774993_7577497 del } \\
\text { CCTGA }\end{array}$ & & NA \\
\hline XRCC4 & frameshift deletion & NM_003401 & c.810delA & p.R270fs & NA \\
\hline XRCC6 & splicing & NM_001469 & c. $589+1 G>T$ & & NA \\
\hline
\end{tabular}




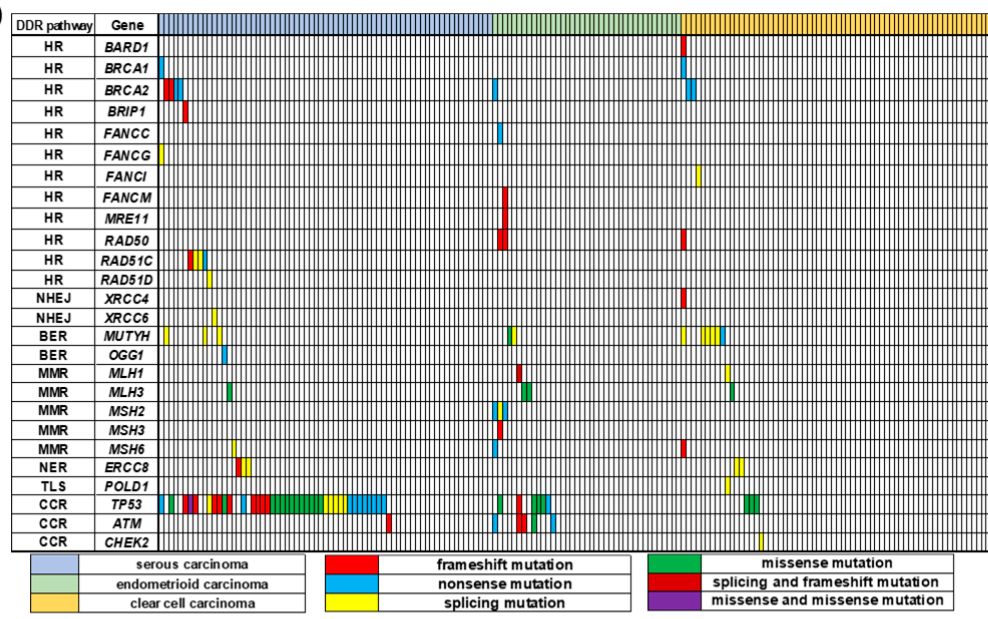

(B)

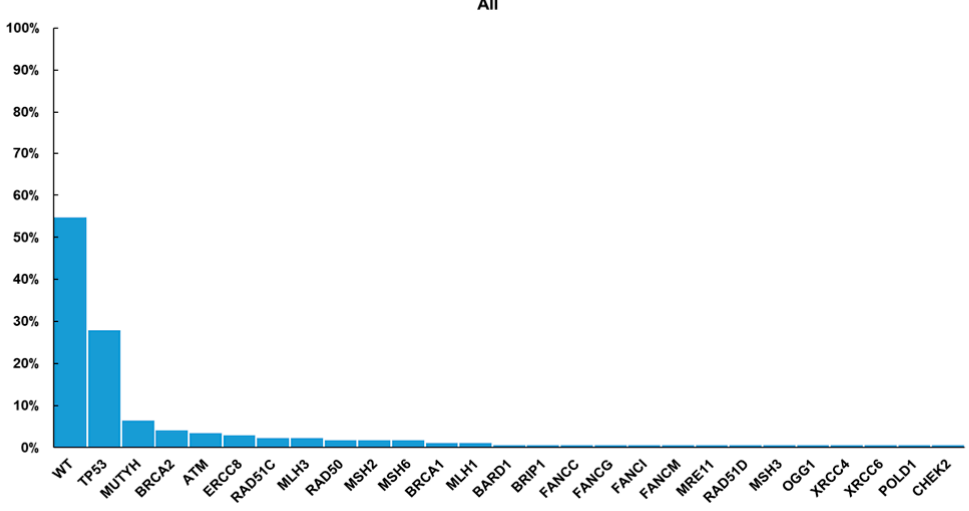

(c)

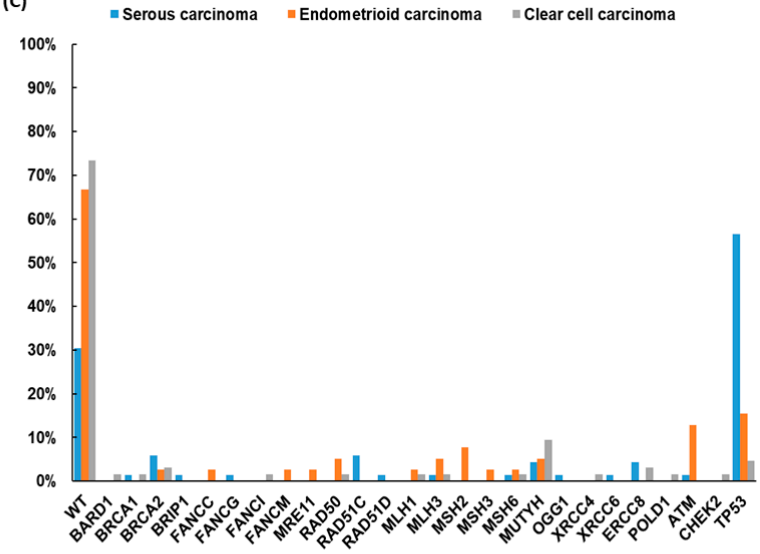

(D)

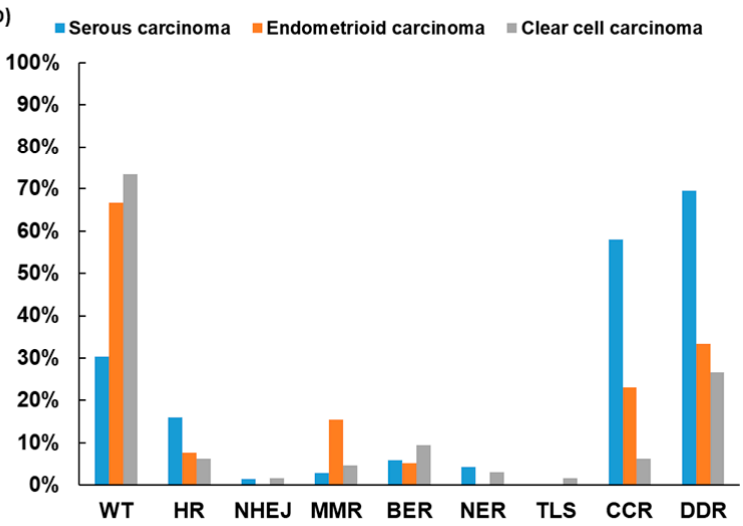

Figure 1. Deleterious DNA damage response (DDR) gene mutations in 172 epithelial ovarian carcinoma (EOC) patients (A) The pattern of DDR mutations of different histologic subtypes. (B) The percentages of DDR mutations in all 172 EOC patients. (C) The percentages of DDR mutations in different histologic subtypes. (D) The percentages of DDR mutations classified by different pathways in different histologic subtypes.

\subsection{Correlation of DDR Gene Mutations with Clinical Outcomes of the EOC Patients}

We evaluated the correlations between the mutation of DDR genes, the clinicopathologic parameters and outcome of the EOC patients. As shown in Table 4, type II tumors had a higher percentage of HR gene mutations than type I tumors (16.18 vs. 6.73\%, $p=0.048$, chi-squared test). Endometrioid carcinoma (15.38\%) had a higher percentage of MMR mutations than those of serous carcinoma $(2.90 \%)$ and clear cell carcinoma $(4.69 \%)(p=0.03$, chi-squared test). Low-grade tumors had a higher percentage of MMR mutations compared with high-grade tumors (17.24 vs. $4.20 \%, p=0.009$, chi-squared test). Type II tumors had a higher percentage of DSBR mutations than type I tumors (17.65 vs. $6.73 \%, p=0.026$, chi-squared test). Serous carcinoma (57.97\%) had a higher percentage of CCR mutations than those of endometrioid carcinoma (23.08\%) and clear cell carcinoma $(6.25 \%)(p<0.001$, chi-squared test). Type II tumors had higher percentage of CCR mutations than those of type I tumors ( 58.82 vs. $12.50 \%, p<0.001$, chi-squared test). The advanced-stage patients had a higher percentage of CCR mutations than the early-stage patients ( 42.72 vs. $13.04 \%$, $p<0.001$, chi-squared test). The recurrent patients had a higher percentage of CCR mutations than those without recurrence (39.22\% vs. $18.57 \%, p=0.004$, chi-squared test). Patients who died of EOC had higher percentages of CCR mutations than living patients (40.79 vs. $22.92 \%, p=0.012$, chi-squared test). Serous carcinoma (69.57\%) had higher percentage of DDR mutations than those of endometrioid carcinoma (33.33\%) and clear cell carcinoma $(26.56 \%)(p<0.001$, chi-squared test). Type II tumors had a higher percentage of DDR mutations than type I tumors (70.59 vs. $28.85 \%, p<0.001$, chi-squared test). The advanced 
stage patients had higher percentage of DDR mutations than the early-stage patients (57.28 vs. $27.54 \%, p<0.001$, chi-squared test). Recurring patients had a higher percentage of DDR mutations than those without recurrence (53.92 vs. $32.86 \%, p=0.006$, chi-squared test). Patients who died of EOC had a higher percentage of DDR mutations than living patients (59.21 vs. $34.38 \%, p=0.001$, chi-squared test).

EOC patients without DDR gene mutation had longer progression-free survival (PFS) $(p=0.0072$, log-rank test, Figure 2A) and overall survival (OS) $(p=0.022, \log$-rank test, Figure $2 \mathrm{~B}$ ) than those with $1 \mathrm{DDR}$ or $\geq 2$ DDR mutations. In serous carcinoma, patients with or without DDR mutations had similar PFS ( $p=0.56$, log-rank test, Figure $2 \mathrm{C})$. Patients with $\geq 2$ DDR mutations had a trend of better OS than those with 1 mutation or none, but it was not statistically significant ( $p=0.47$, log-rank test, Figure 2D). In endometrioid carcinoma, patients with $\geq 2$ DDR gene mutations had shorter PFS ( $p=0.0035$, log-rank test, Figure $2 \mathrm{E}$ ) and OS ( $p=0.015$, log-rank test, Figure $2 \mathrm{~F}$ ) than those with 1 mutation or none. In clear cell carcinoma, patients with $\geq 2$ DDR gene mutations had significantly shorter PFS ( $p=0.0056$, log-rank test, Figure $2 \mathrm{G})$ and OS ( $p=0.0046, \log$-rank test, Figure $2 \mathrm{H})$ than those with 1 DDR mutation or none.

Tumor recurrence with CCR gene mutation (HR: $1.68(1.12-2.50), p=0.011), 1$ DDR gene mutation (HR: $1.71(1.12-2.60), p=0.013)$, endometrioid carcinoma (HR: $0.17(0.08-0.37)$, $p<0.001)$, type II tumor (HR: 2.69 (1.81-4.00), $p<0.001)$, advanced-stage carcinoma (HR: 5.29 (3.16-8.85), $p<0.001)$, high-grade tumor (HR: 5.57 (2.26-13.70), $p<0.001)$ and optimal debulking surgery (HR: $0.28(0.18-0.41), p<0.001)$ were significant in the univariate Cox regression model (Table 5). Advanced-stage carcinoma (HR: 3.08 (1.63-5.80), $p=0.001$ ) and optimal debulking surgery (HR: $0.51(0.32-0.80), p=0.004)$ were important prognostic factors in the multivariate analysis. Cancer-related death with TLS gene mutation (HR: 33.76 (3.95-289.00), $p=0.001), 1$ DDR gene mutation (HR: 1.96 (1.20-3.20), $p=0.007$ ), endometrioid carcinoma (HR: $0.12(0.04-0.38), p<0.001)$, type II tumor (HR: 1.88 (1.19-2.96), $p=0.007)$, advanced-stage carcinoma (HR: $6.84(3.28-14.25), p<0.001)$, high-grade tumor (HR: 17.97 (2.50-129.29), $p=0.004)$ and optimal debulking surgery (HR: $0.26(0.16-0.41)$, $p<0.001)$ were significant in the univariate Cox regression model. Type II tumor (HR: 0.35 (0.20-0.60), $p<0.001)$, TLS gene mutation (HR: 9.57 (1.08-84.83), $p=0.042)$, advanced-stage carcinoma (HR: 4.82 (2.09-11.09), $p<0.001)$ and optimal debulking surgery (HR: 0.38 $(0.22-0.64), p<0.001)$ were important prognostic factors in the multivariate analysis. 
Table 4. The correlation of DDR gene mutations with clinical parameters in the epithelial ovarian cancer patients.

\begin{tabular}{|c|c|c|c|c|c|c|c|c|c|c|c|c|c|c|}
\hline \multirow[t]{2}{*}{ Genes } & & \multicolumn{3}{|c|}{ Histology } & \multicolumn{2}{|c|}{ Type } & \multicolumn{2}{|c|}{ FIGO Stage } & \multicolumn{2}{|c|}{ Tumor Grade } & \multicolumn{2}{|c|}{ Recurrence } & \multicolumn{2}{|c|}{ Death } \\
\hline & & OSA & OEA & OCCA & I & II & Early & Advanced & Low & High & No & Yes & No & Yes \\
\hline Total & 172 & 69 & 39 & 64 & 104 & 68 & 69 & 103 & 29 & 143 & 70 & 102 & 96 & 76 \\
\hline Wild type & 154 & 58 & 36 & 60 & 97 & 57 & 64 & 90 & 26 & 128 & 64 & 90 & 89 & 65 \\
\hline & $89.53 \%$ & $84.06 \%$ & $92.31 \%$ & $93.75 \%$ & $93.27 \%$ & $83.82 \%$ & $92.75 \%$ & $87.38 \%$ & $89.66 \%$ & $89.51 \%$ & $91.43 \%$ & $88.24 \%$ & $92.71 \%$ & $85.53 \%$ \\
\hline Mutation & 18 & 11 & 3 & 4 & 7 & 11 & 5 & 13 & 3 & 15 & 6 & 12 & 7 & 11 \\
\hline & $10.47 \%$ & $15.94 \%$ & $7.69 \%$ & $6.25 \%$ & $6.73 \%$ & $16.18 \%$ & $7.25 \%$ & $12.62 \%$ & $10.34 \%$ & $10.49 \%$ & $8.57 \%$ & $11.76 \%$ & $7.29 \%$ & $14.47 \%$ \\
\hline$p$ value * & & & & 0.154 & & 0.048 & & 0.259 & & 0.981 & & 0.502 & & 0.126 \\
\hline \multicolumn{15}{|l|}{ NHEJ } \\
\hline Wild type & 170 & 68 & 39 & 63 & 103 & 67 & 69 & 101 & 29 & 141 & 70 & 100 & 96 & 74 \\
\hline & $98.84 \%$ & $98.55 \%$ & $100.00 \%$ & $98.44 \%$ & $99.04 \%$ & $98.53 \%$ & $100.00 \%$ & $98.06 \%$ & $100.00 \%$ & $98.60 \%$ & $100.00 \%$ & $98.04 \%$ & $100.00 \%$ & $97.37 \%$ \\
\hline Mutation & 2 & 1 & 0 & 1 & 1 & 1 & 0 & 2 & 0 & 2 & 0 & 2 & 0 & 2 \\
\hline & $1.16 \%$ & $1.45 \%$ & $0.00 \%$ & $1.56 \%$ & $0.96 \%$ & $1.47 \%$ & $0.00 \%$ & $1.94 \%$ & $0.00 \%$ & $1.40 \%$ & $0.00 \%$ & $1.96 \%$ & $0.00 \%$ & $2.63 \%$ \\
\hline$p$ value* & & & & 0.742 & & 0.761 & & 0.244 & & 0.522 & & 0.239 & & 0.11 \\
\hline \multicolumn{15}{|l|}{ MMR } \\
\hline & $93.60 \%$ & $97.10 \%$ & $84.62 \%$ & $95.31 \%$ & $91.35 \%$ & $97.06 \%$ & $94.20 \%$ & $93.20 \%$ & $82.76 \%$ & $95.80 \%$ & $94.29 \%$ & $93.14 \%$ & $94.79 \%$ & $92.11 \%$ \\
\hline Mutation & 11 & 2 & 6 & 3 & 9 & 2 & 4 & 7 & 5 & 6 & 4 & 7 & 5 & 6 \\
\hline & $6.40 \%$ & $2.90 \%$ & $15.38 \%$ & $4.69 \%$ & $8.65 \%$ & $2.94 \%$ & $5.80 \%$ & $6.80 \%$ & $17.24 \%$ & $4.20 \%$ & $5.71 \%$ & $6.86 \%$ & $5.21 \%$ & $7.89 \%$ \\
\hline$p$ value * & & & & 0.03 & & 0.134 & & 0.793 & & 0.009 & & 0.762 & & 0.475 \\
\hline \multicolumn{15}{|l|}{ BER } \\
\hline Wild type & 160 & 65 & 37 & 58 & 96 & 64 & 65 & 95 & 27 & 133 & 66 & 94 & 91 & 69 \\
\hline & $93.02 \%$ & $94.20 \%$ & $94.87 \%$ & $90.63 \%$ & $92.31 \%$ & $94.12 \%$ & $94.20 \%$ & $92.23 \%$ & $93.10 \%$ & $93.01 \%$ & $94.29 \%$ & $92.16 \%$ & $94.79 \%$ & $90.79 \%$ \\
\hline Mutation & 12 & 4 & 2 & 6 & 8 & 4 & 4 & 8 & 2 & 10 & 4 & 8 & 5 & 7 \\
\hline & $6.98 \%$ & $5.80 \%$ & $5.13 \%$ & $9.38 \%$ & $7.69 \%$ & $5.88 \%$ & $5.80 \%$ & $7.77 \%$ & $6.90 \%$ & $6.99 \%$ & $5.71 \%$ & $7.84 \%$ & $5.21 \%$ & $9.21 \%$ \\
\hline$p$ value $*$ & & & & 0.631 & & 0.649 & & 0.619 & & 0.985 & & 0.59 & & 0.306 \\
\hline
\end{tabular}


Table 4. Cont

\begin{tabular}{|c|c|c|c|c|c|c|c|c|c|c|c|c|c|c|}
\hline \multirow[t]{2}{*}{ Genes } & & \multicolumn{3}{|c|}{ Histology } & \multicolumn{2}{|c|}{ Type } & \multicolumn{2}{|c|}{ FIGO Stage } & \multicolumn{2}{|c|}{ Tumor Grade } & \multicolumn{2}{|c|}{ Recurrence } & \multicolumn{2}{|c|}{ Death } \\
\hline & & OSA & OEA & OCCA & $\mathbf{I}$ & II & Early & Advanced & Low & High & No & Yes & No & Yes \\
\hline \multicolumn{15}{|l|}{ NER } \\
\hline & $97.09 \%$ & $95.65 \%$ & $100.00 \%$ & $96.88 \%$ & $98.08 \%$ & $95.59 \%$ & $97.10 \%$ & $97.09 \%$ & $100.00 \%$ & $96.50 \%$ & $95.71 \%$ & $98.04 \%$ & $96.88 \%$ & $97.37 \%$ \\
\hline Mutation & 5 & 3 & 0 & 2 & 2 & 3 & 2 & 3 & 0 & 5 & 3 & 2 & 3 & 2 \\
\hline & $2.91 \%$ & $4.35 \%$ & $0.00 \%$ & $3.13 \%$ & $1.92 \%$ & $4.41 \%$ & $2.90 \%$ & $2.91 \%$ & $0.00 \%$ & $3.50 \%$ & $4.29 \%$ & $1.96 \%$ & $3.13 \%$ & $2.63 \%$ \\
\hline$p$ value $*$ & & & & 0.43 & & 0.342 & & 0.996 & & 0.307 & & 0.373 & & 0.848 \\
\hline \multicolumn{15}{|l|}{ TLS } \\
\hline Wild type & 171 & 69 & 39 & 63 & 103 & 68 & 69 & 102 & 29 & 142 & 70 & 101 & 96 & 75 \\
\hline & $99.42 \%$ & $100.00 \%$ & $100.00 \%$ & $98.44 \%$ & $99.04 \%$ & $100.00 \%$ & $100.00 \%$ & $99.03 \%$ & $100.00 \%$ & $99.30 \%$ & $100.00 \%$ & $99.02 \%$ & $100.00 \%$ & $98.68 \%$ \\
\hline Mutation & 1 & 0 & 0 & 1 & 1 & 0 & 0 & 1 & 0 & 1 & 0 & 1 & 0 & 1 \\
\hline & $0.58 \%$ & $0.00 \%$ & $0.00 \%$ & $1.56 \%$ & $0.96 \%$ & $0.00 \%$ & $0.00 \%$ & $0.97 \%$ & $0.00 \%$ & $0.70 \%$ & $0.00 \%$ & $0.98 \%$ & $0.00 \%$ & $1.32 \%$ \\
\hline$p$ value $*$ & & & & 0.428 & & 0.417 & & 0.412 & & 0.652 & & 0.406 & & 0.26 \\
\hline \multicolumn{15}{|l|}{ DSBR } \\
\hline Wild type & 153 & 57 & 36 & 60 & 97 & 56 & 64 & 89 & 26 & 127 & 64 & 89 & 89 & 64 \\
\hline & $11.05 \%$ & $17.39 \%$ & $7.69 \%$ & $6.25 \%$ & $6.73 \%$ & $17.65 \%$ & $7.25 \%$ & $13.59 \%$ & $10.34 \%$ & $11.19 \%$ & $8.57 \%$ & $12.75 \%$ & $7.29 \%$ & $15.79 \%$ \\
\hline$p$ value $*$ & & & & 0.092 & & 0.026 & & 0.193 & & 0.895 & & 0.391 & & 0.077 \\
\hline \multicolumn{15}{|l|}{ SSBR } \\
\hline Wild type & 145 & 60 & 31 & 54 & 86 & 59 & 59 & 86 & 22 & 123 & 59 & 86 & 83 & 62 \\
\hline & $84.30 \%$ & $86.96 \%$ & $79.49 \%$ & $84.38 \%$ & $82.69 \%$ & $86.76 \%$ & $85.51 \%$ & $83.50 \%$ & $75.86 \%$ & $86.01 \%$ & $84.29 \%$ & $84.31 \%$ & $86.46 \%$ & $81.58 \%$ \\
\hline Mutation & 27 & 9 & 8 & 10 & 18 & 9 & 10 & 17 & 7 & 20 & 11 & 16 & 13 & 14 \\
\hline & $15.70 \%$ & $13.04 \%$ & $20.51 \%$ & $15.63 \%$ & $17.31 \%$ & $13.24 \%$ & $14.49 \%$ & $16.50 \%$ & $24.14 \%$ & $13.99 \%$ & $15.71 \%$ & $15.69 \%$ & $13.54 \%$ & $18.42 \%$ \\
\hline$p$ value $*$ & & & & 0.591 & & 0.473 & & 0.722 & & 0.171 & & 0.996 & & 0.382 \\
\hline \multicolumn{15}{|l|}{ CCR } \\
\hline Wild type & 119 & 29 & 30 & 60 & 91 & 28 & 60 & 59 & 24 & 95 & 57 & 62 & 74 & 45 \\
\hline & $69.19 \%$ & $42.03 \%$ & $76.92 \%$ & $93.75 \%$ & $87.50 \%$ & $41.18 \%$ & $86.96 \%$ & $57.28 \%$ & $82.76 \%$ & $66.43 \%$ & $81.43 \%$ & $60.78 \%$ & $77.08 \%$ & $59.21 \%$ \\
\hline Mutation & 53 & 40 & 9 & 4 & 13 & 40 & 9 & 44 & 5 & 48 & 13 & 40 & 22 & 31 \\
\hline & $30.81 \%$ & $57.97 \%$ & $23.08 \%$ & $6.25 \%$ & $12.50 \%$ & $58.82 \%$ & $13.04 \%$ & $42.72 \%$ & $17.24 \%$ & $33.57 \%$ & $18.57 \%$ & $39.22 \%$ & $22.92 \%$ & $40.79 \%$ \\
\hline$p$ value $*$ & & & & $<0.001$ & & $<0.001$ & & $<0.001$ & & 0.083 & & 0.004 & & 0.012 \\
\hline
\end{tabular}


Table 4. Cont

\begin{tabular}{|c|c|c|c|c|c|c|c|c|c|c|c|c|c|c|}
\hline \multirow[t]{2}{*}{ Genes } & & \multicolumn{3}{|c|}{ Histology } & \multicolumn{2}{|c|}{ Type } & \multicolumn{2}{|c|}{ FIGO Stage } & \multicolumn{2}{|c|}{ Tumor Grade } & \multicolumn{2}{|c|}{ Recurrence } & \multicolumn{2}{|c|}{ Death } \\
\hline & & OSA & OEA & OCCA & I & II & Early & Advanced & Low & High & No & Yes & No & Yes \\
\hline \multicolumn{15}{|l|}{ DDR } \\
\hline Wild type & $54.65 \%$ & $30.43 \%$ & $66.67 \%$ & $73.44 \%$ & $71.15 \%$ & $29.41 \%$ & $72.46 \%$ & $42.72 \%$ & $68.97 \%$ & $51.75 \%$ & $67.14 \%$ & $46.08 \%$ & $65.63 \%$ & $40.79 \%$ \\
\hline 1 gene & 57 & 35 & 7 & 15 & 22 & 35 & 14 & 43 & 5 & 52 & 16 & 41 & 24 & 33 \\
\hline mutation & $33.14 \%$ & $50.72 \%$ & $17.95 \%$ & $23.44 \%$ & $21.15 \%$ & $51.47 \%$ & $20.29 \%$ & $41.75 \%$ & $17.24 \%$ & $36.36 \%$ & $22.86 \%$ & $40.20 \%$ & $25.00 \%$ & $43.42 \%$ \\
\hline 2 gene & 15 & 12 & 2 & 1 & 3 & 12 & 2 & 13 & 0 & 15 & 4 & 11 & 6 & 9 \\
\hline 3 gene & 2 & 1 & 1 & 0 & 1 & 1 & 1 & 1 & 1 & 1 & 1 & 1 & 1 & 1 \\
\hline mutations & $1.16 \%$ & $1.45 \%$ & $2.56 \%$ & $0.00 \%$ & $0.96 \%$ & $1.47 \%$ & $1.45 \%$ & $0.97 \%$ & $3.45 \%$ & $0.70 \%$ & $1.43 \%$ & $0.98 \%$ & $1.04 \%$ & $1.32 \%$ \\
\hline 4 gene & 2 & 0 & 2 & 0 & 2 & 0 & 2 & 0 & 2 & 0 & 2 & 0 & 2 & 0 \\
\hline mutations & $1.16 \%$ & $0.00 \%$ & $5.13 \%$ & $0.00 \%$ & $1.92 \%$ & $0.00 \%$ & $2.90 \%$ & $0.00 \%$ & $6.90 \%$ & $0.00 \%$ & $2.86 \%$ & $0.00 \%$ & $2.08 \%$ & $0.00 \%$ \\
\hline 5 gene & 1 & 0 & 1 & 0 & 1 & 0 & 0 & 1 & 1 & 0 & 0 & 1 & 0 & 1 \\
\hline Mutations & $0.58 \%$ & $0.00 \%$ & $2.56 \%$ & $0.00 \%$ & $0.96 \%$ & $0.00 \%$ & $0.00 \%$ & $0.97 \%$ & $3.45 \%$ & $0.00 \%$ & $0.00 \%$ & $0.98 \%$ & $0.00 \%$ & $1.32 \%$ \\
\hline 6 gene & 1 & 0 & 0 & 1 & 1 & 0 & 0 & 1 & 0 & 1 & 0 & 1 & 0 & 1 \\
\hline mutations & $0.58 \%$ & $0.00 \%$ & $0.00 \%$ & $1.56 \%$ & $0.96 \%$ & $0.00 \%$ & $0.00 \%$ & $0.97 \%$ & $0.00 \%$ & $0.70 \%$ & $0.00 \%$ & $0.98 \%$ & $0.00 \%$ & $1.32 \%$ \\
\hline Total & 78 & 48 & 13 & 17 & 30 & 48 & 19 & 59 & 9 & 69 & 23 & 55 & 33 & 45 \\
\hline$p$ value * & & & & $<0.001$ & & $<0.001$ & & $<0.001$ & & 0.089 & & 0.006 & & 0.001 \\
\hline
\end{tabular}

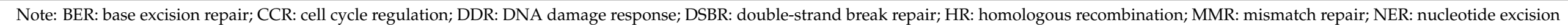

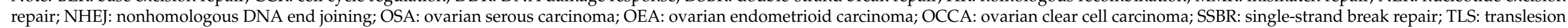
synthesis. * Pearson's chi-squared test 
(A) PFS of all EOC patients

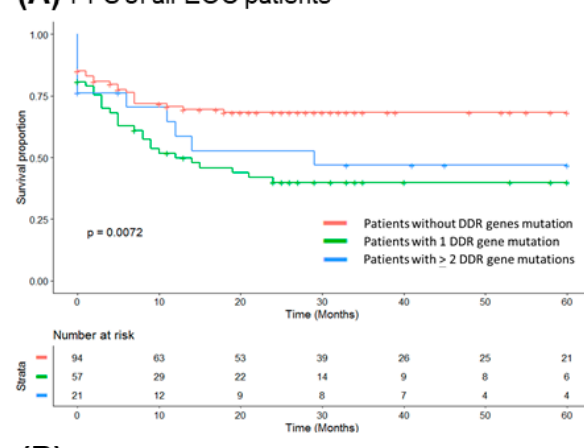

(B) OS of all EOC patients
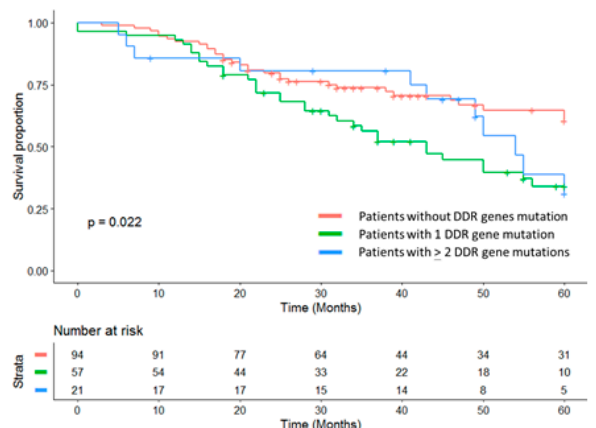

(C) PFS of serous carcinoma patients

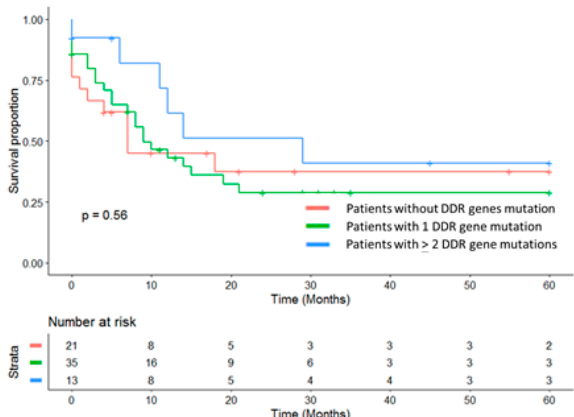

(D) OS of serous carcinoma patients

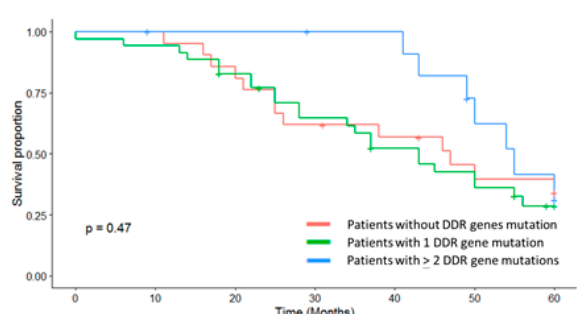

(E) PFS of endometrioid carcinoma patients

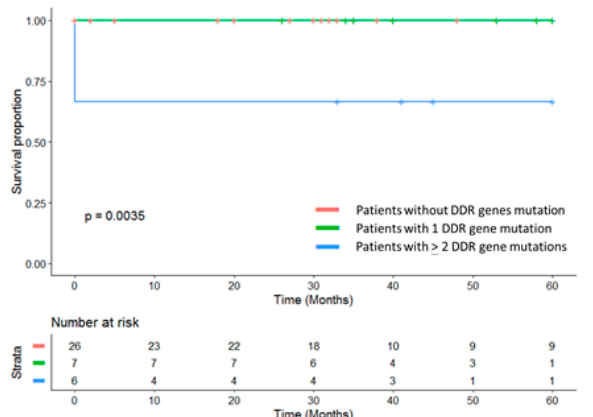

(F) OS of endometrioid carcinoma patients

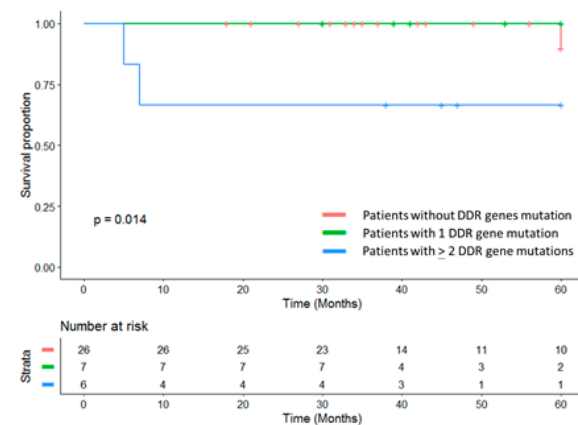

(G) PFS of clear cell carcinoma patients
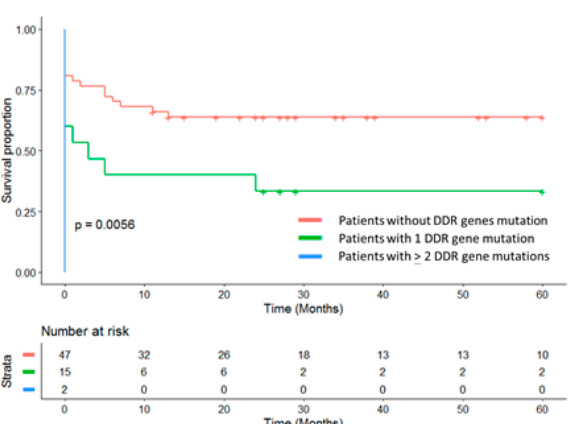

(H) OS of clear cell carcinoma patients

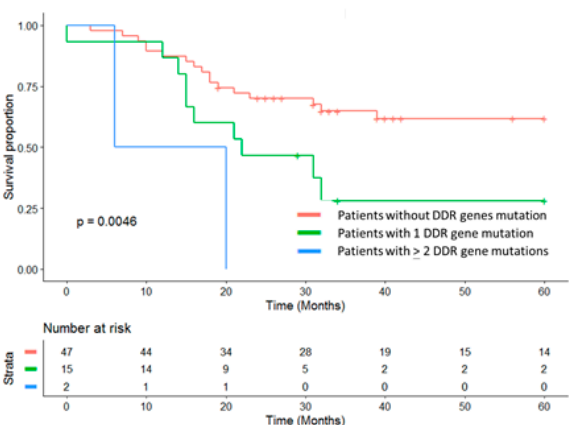

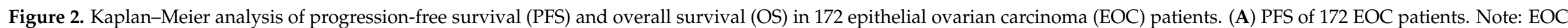

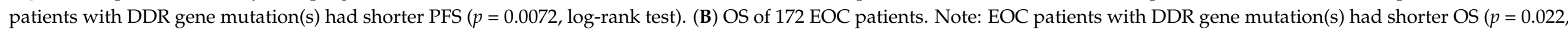

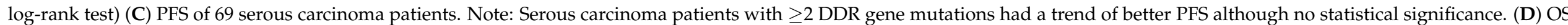

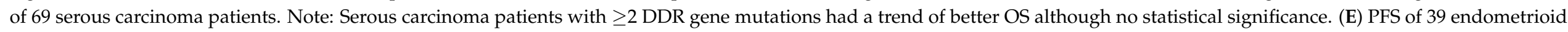

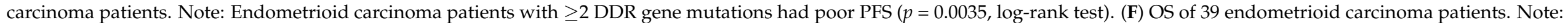

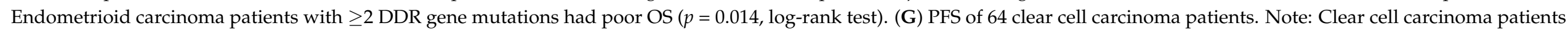

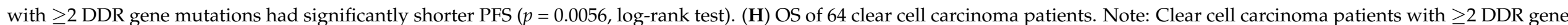
mutations had significantly shorter OS ( $p=0.0046$, log-rank test). 
Table 5. Cox regression model for the risk factors for recurrence and death in all patients $(n=172)$.

\begin{tabular}{|c|c|c|c|c|c|c|c|c|c|}
\hline \multirow[t]{2}{*}{ Factors } & & \multicolumn{4}{|c|}{ Recurrence } & \multicolumn{4}{|c|}{ Death } \\
\hline & & Univariate & & Multivariate & & Univariate & & Multivariate & \\
\hline & $\mathbf{n}$ & $\begin{array}{c}\text { Hazard Ratio } \\
(95 \% \text { CI })\end{array}$ & $p$ & $\begin{array}{l}\text { Hazard Ratio } \\
\quad(95 \% \mathrm{CI})\end{array}$ & $p$ & $\begin{array}{c}\text { Hazard Ratio } \\
(95 \% \text { CI })\end{array}$ & $p$ & $\begin{array}{c}\text { Hazard Ratio } \\
(95 \% \text { CI })\end{array}$ & $p$ \\
\hline \multicolumn{10}{|l|}{ Histology } \\
\hline OSA & 69 & 1 (reference) & & 1 (reference) & & 1 (reference) & & 1 (reference) & \\
\hline OEA & 39 & $0.17(0.08-0.37)$ & $<0.001$ & $0.42(0.16-1.12)$ & 0.082 & $0.12(0.04-0.38)$ & $<0.001$ & $0.45(0.13-1.55)$ & 0.205 \\
\hline OCCA & 64 & $0.96(0.64-1.44)$ & 0.835 & & & $1.37(0.86-2.18)$ & 0.188 & & \\
\hline I & 104 & 1 (reference) & & 1 (reference) & & 1 (reference) & & 1 (reference) & \\
\hline II & 68 & $2.69(1.81-4.00)$ & $<0.001$ & $0.77(0.46-1.28)$ & 0.311 & $1.88(1.19-2.96)$ & 0.007 & $0.35(0.20-0.60)$ & $<0.001$ \\
\hline \multicolumn{10}{|l|}{ FIGO stage } \\
\hline Early & 69 & 1 (reference) & & 1 (reference) & & 1 (reference) & & 1 (reference) & \\
\hline Advanced & 103 & $5.29(3.16-8.85)$ & $<0.001$ & $3.08(1.63-5.80)$ & 0.001 & $6.84(3.28-14.25)$ & $<0.001$ & $4.82(2.09-11.09)$ & $<0.001$ \\
\hline \multicolumn{10}{|l|}{ Tumor grade } \\
\hline Low & 29 & 1 (reference) & & 1 (reference) & & 1 (reference) & & 1 (reference) & \\
\hline High & 143 & $5.57(2.26-13.70)$ & $<0.001$ & $1.68(0.55-5.15)$ & 0.366 & $\begin{array}{c}17.97 \\
(2.50-129.29)\end{array}$ & 0.004 & $7.38(0.93-58.28)$ & 0.058 \\
\hline \multicolumn{10}{|c|}{ Debulking surgery } \\
\hline Suboptimal & 60 & 1 (reference) & & 1 (reference) & & 1 (reference) & & 1 (reference) & \\
\hline Optimal & 112 & $0.28(0.18-0.41)$ & $<0.001$ & $0.51(0.32-0.80)$ & 0.004 & $0.26(0.16-0.41)$ & $<0.001$ & $0.38(0.22-0.64)$ & $<0.001$ \\
\hline \multicolumn{10}{|l|}{ HR } \\
\hline Wild type & 154 & 1 (reference) & & & & 1 (reference) & & & \\
\hline Mutation & 18 & $1.22(0.67-2.23)$ & 0.516 & & & $1.15(0.59-2.25)$ & 0.674 & & \\
\hline \multicolumn{10}{|l|}{ NHEJ } \\
\hline Wild type & 170 & 1 (reference) & & & & 1 (reference) & & & \\
\hline Mutation & 2 & $2.04(0.50-8.28)$ & 0.319 & & & $2.52(0.62-10.32)$ & 0.197 & & \\
\hline \multicolumn{10}{|l|}{ MMR } \\
\hline Wild type & 161 & 1 (reference) & & & & 1 (reference) & & & \\
\hline Mutation & 11 & $1.31(0.61-2.83)$ & 0.487 & & & $1.88(0.81-4.33)$ & 0.139 & & \\
\hline
\end{tabular}


Table 5. Cont.

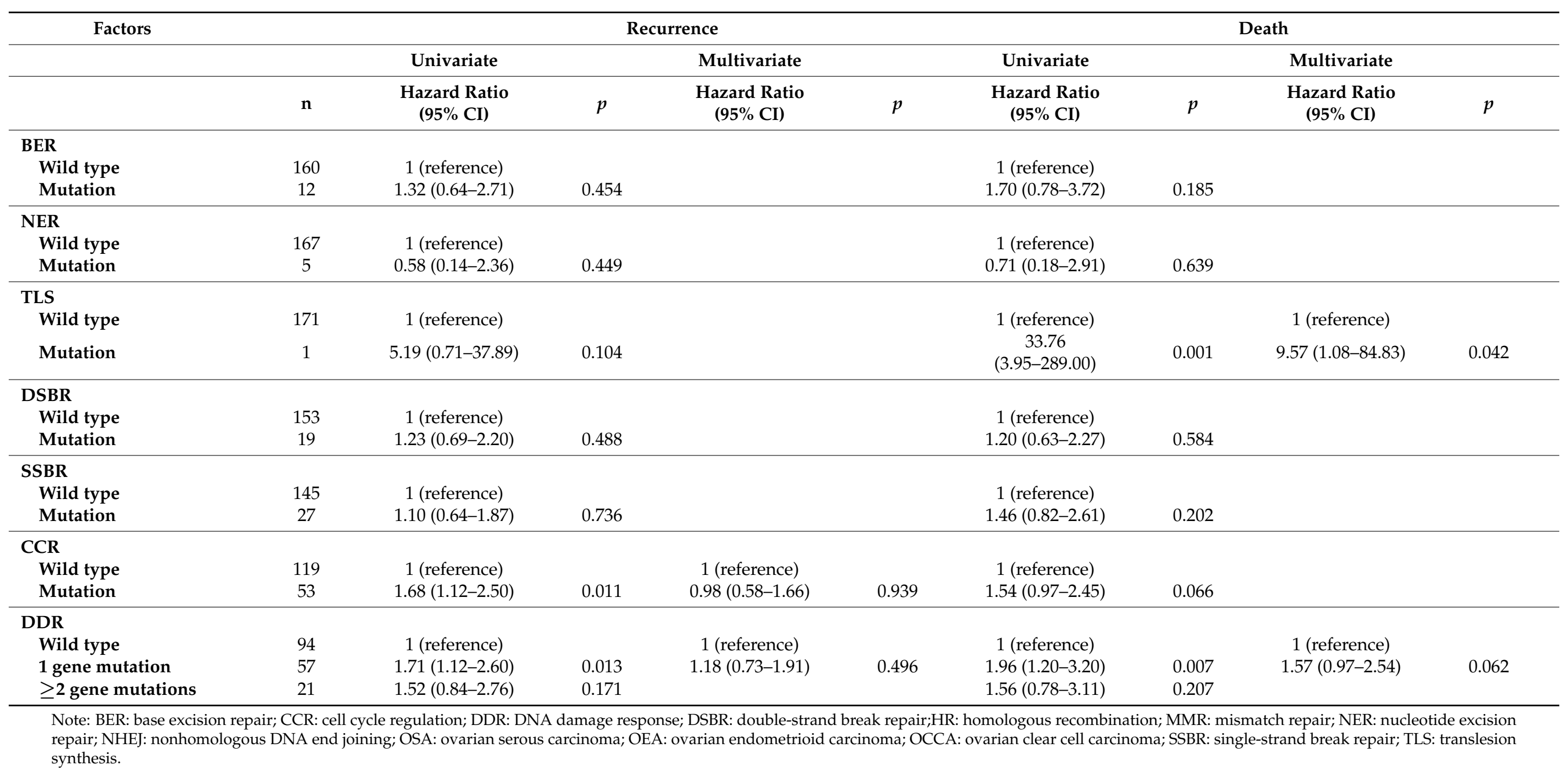




\section{Discussion}

Our study showed that nearly half of the epithelial ovarian cancer (EOC) patients had DNA damage response (DDR) gene mutations with varied proportions of histological subtypes. Two-thirds of serous adenocarcinoma patients, one-third of endometrioid adenocarcinoma patients and one-fourth of clear cell carcinoma patients had DDR gene mutations. Our DDR gene panel consisted of the genes involved in single-strand break repair, double-strand break repair and cell cycle regulation, including the genes recommended by National Comprehensive Cancer Network (NCCN) guidelines as cost-effective tools for assessing the lifetime risk of EOC, such as ATM, BRCA1/2, BRIP1, MLH1, MSH2, MSH6, PALB2, RAD51C and RAD51D [32]. The major components of DDR gene mutations were CCR in serous, CCR and SSBR in endometrioid and SSBR in clear cell carcinomas; CCR and DSBR in type II tumors (high-grade serous carcinoma in the cohort); and SSBR in type I tumors. A multiple DDR gene panel increased the detection rate of somatic mutation of genes involved in DNA damage repair pathway in comparison with a BRCA test alone. The percentage of $B R C A 1 / 2$ somatic mutation in serous carcinoma was 7.2 , which was compatible with the $6-7 \%$ in previous studies [33-37]. The non-BRCA HR somatic mutation of our study was more than $10 \%$ in serous and endometrioid carcinomas, and the MMR somatic mutation was around $15 \%$ in endometrioid carcinomas, which was compatible with the previous study [38].

Our study showed that ovarian clear cell carcinoma patients with DDR gene mutations had an unfavorable survival prognosis. Those who had somatic DDR mutations were significantly associated with advanced-stage carcinomas, tumor recurrence and tumorrelated death. The trend was different in histological subtypes as serous carcinomas or type II tumors with DDR mutation showed a better survival trend. Non-serous or type I EOC patients with DDR mutations had a poor prognosis, especially in clear cell carcinoma. Ovarian clear cell carcinoma is an aggressive drug-resistant subtype of EOC in association with endometriosis and glycogen accumulation. It accounts for about $5-13 \%$ of all EOCs in Western populations, but up to $20-25 \%$ in East Asia, including Taiwan [2,3]. Previous studies showed that the somatic mutations of ovarian clear cell carcinoma (mainly in ARID1A, PIK3CA, KRAS and PPP2R1A) might be related to chromatin remodeling, cell proliferation, cell cycle checkpointing and cytoskeletal organization [39-49]. However, the frequent mutations of ARID1A, PIK3CA, PPP2R1A or TP53 in ovarian clear cell carcinoma did not correlate well with the prognosis [45]. Other infrequent gene mutations of clear cell carcinoma included ARID1B, ARID3A, CREBBP, CSMD3, CTNNB1, LPHN3, LRP1B, MAGEE1, MLH1, MLL3, MUC4, PIK3R1, PTEN and TP53 [41,43,46,48,49]. DDR gene mutations in ovarian clear cell carcinoma was unclear in the literature, and our finding of an unfavorable prognosis in clear cell carcinoma patients with DDR gene mutations could provide useful information.

Our DDR gene panel could provide a scientific rationale for patient selection in future clinical trials that target DNA damage repair response pathways, especially in clear cell carcinoma. BRCA gene tests or companion HRD assays are currently suggested for PARPi, but there are unmet problems that need to be resolved [11-15,20]. The most important one is that the HRD assays cannot consistently identify patients who do not benefit from PARPi therapy. The consensus for the cut-off value was indeterminate because the thresholds of HRD assays were developed from retrospective exploratory analyses [11,50,51]. Generally, advance-stage, high-grade serous carcinoma patients with tumor $B R C A(\mathrm{t} B R C A)$ mutations, including germline ( $\mathrm{g} B R C A$ ) or somatic ( $\mathrm{s} B R C A$ ), derived the greatest benefit from PARPi maintenance therapy [11-15]. Approximately $11-18 \%$ of patients had a gBRCA mutation, and another $6-7 \%$ patients had an sBRCA mutation with a negative $\mathrm{g} B R C A$ test [33-37]. However, about $5 \%$ of gBRCA mutated patients tested negative for $\mathrm{t} B R C A$ [52-54]. The non- $B R C A$ HR gene mutations were usually pooled together to interpret the association with clinical outcomes in previous studies because of their relatively low prevalence [35,55-57]. Twenty-one platinum-sensitive recurrent patients with non$B R C A$ somatic mutations (BRIP1, CDK12, RAD54L and RAD51B) derived benefit from 
olaparib in study 19 [58]. In ARIEL2, there were 20 patients with non-BRCA HR gene mutations (ATM, BRIP1, CHEK2, FANCA, FANCI, FANCM, NBN, RAD51B, RAD51C and $R A D 54 L$ ), but the sensitivity in discriminating a rucaparib response was only $11 \%$ [59]. However, $B R C A$ wild type EOC patients still benefitted from PARPi, which indicated that a $B R C A$ test by itself was inadequate for selecting EOC patients for PARPi [13-15]. It needs to be determined which individual or panel of non-BRCA HR genes could be used to predict a PARPi response, especially in non-serous EOC patients.

There were limitations to our study. First, germline gene mutations were not investigated. These not only inform the patients but also identify family members of the possible risk of malignancy [52-54]. The NCCN suggested germline gene tests of $A T M, B R C A 1 / 2$, BRIP1, MLH1, MSH2, MSH6, PALB2, RAD51C, RAD51D and STK11 to assess the lifetime risk of EOC [32], but how many genes should be included in the panel is inconclusive. Second, the numerous variants of uncertain significance (VUSs) identified by multiple gene panels would cause controversy in risk assessment and management [60-62]. The biological functions and clinical impacts of most individual mutations in the genomic loci have not been well characterized, especially for VUSs [63]. Even in the well-studied $B R C A$ gene, there is a difference among laboratories in the VUS reporting rate (3-50\%), detection protocols and management strategies [64]. Further sharing and integration of gene sequencing data in an open database might decrease VUSs. Third, the cohort sample was not large enough; only the trends of clinical prognosis that correlated with each DDR pathway were found. Further large-scale investigations are needed.

\section{Conclusions}

Our study found that nearly half of the EOC patients had DDR gene mutations of varying proportions in the histological subtypes. Patients with somatic DDR mutations were significantly associated with advanced-stage carcinoma, tumor recurrence and tumorrelated death. Type I EOC patients with DDR mutations had an unfavorable prognosis, especially for clear cell carcinoma. A broad multiple-gene DDR panel would provide not only comprehensive information of gene mutations but also a rationale for a future study of a novel therapy target for DNA damage response pathways.

Supplementary Materials: The following are available online at https:/ / www.mdpi.com/article/10 .3390/biomedicines9101384/s1, Figure S1: Variants of uncertain significance (VUS) with the potential of being deleterious mutations, Figure S2: The distribution of all 114 deleterious DDR gene mutations, Table S1: Variants of uncertain significance (VUSs) with the potential of being deleterious mutations in the cohort, Table S2: The percentages of DDR gene mutations in all patients.

Author Contributions: Conceptualization, Y.-C.C., P.-H.L., W.-F.C. and C.-A.C.; methodology, Y.-C.C. and P.-H.L.; software, P.-H.L. and T.-P.L.; validation, Y.-C.C., P.-H.L. and T.-P.L.; formal analysis, Y.-C.C., P.-H.L. and T.-P.L.; investigation, Y.-C.C., Y.-J.T., H.-C.H., C.-Y.W., C.-Y.L., H.S.; resources, Y.-C.C., K.-T.K., Y.-J.T., H.-C.H., C.-Y.W., C.-Y.L., H.S., C.-A.C. and W.-F.C.; data curation, Y.-C.C., P.-H.L. and T.-P.L.; writing-original draft preparation, Y.-C.C. and P.-H.L.; writing-review and editing, C.-A.C. and W.-F.C.; visualization, Y.-C.C. and P.-H.L.; supervision, C.-A.C. and W.-F.C.; funding acquisition, Y.-C.C., C.-A.C. and W.-F.C. All authors have read and agreed to the published version of the manuscript.

Funding: This work was supported by research grants from the National Taiwan University Hospital (NTUH. 105-N02, UN105-059, 108-S4230 and 109-S4570).

Institutional Review Board Statement: The study was conducted according to the guidelines of the Declaration of Helsinki, and approved by the Ethics Committee of National Taiwan University Hospital (201509042RINA, approved on 24 November 2015 and 201608025RINA, approved on 07 October 2016).

Informed Consent Statement: Informed consent was obtained from all subjects involved in the study. 
Data Availability Statement: The datasets generated and/or analyzed during the current study are available from the corresponding author on reasonable request.

Acknowledgments: The authors thank the A1 laboratory of the National Taiwan University Hospital for the Illumina Miseq NGS platform, the National Applied Research Laboratories for providing access of high-performance computer to analyze NGS data, and the 7th Core Laboratory Facility of the Department of Medical Research of National Taiwan University Hospital for supporting the work.

Conflicts of Interest: The authors declare no conflict of interest.

\section{References}

1. Siegel, R.L.; Miller, K.D.; Fuchs, H.E.; Jemal, A. Cancer Statistics, 2021. CA Cancer J. Clin 2021, 71, 7-33. [CrossRef]

2. Torre, L.A.; Trabert, B.; DeSantis, C.E.; Miller, K.D.; Samimi, G.; Runowicz, C.D.; Gaudet, M.M.; Jemal, A.; Siegel, R.L. Ovarian cancer statistics, 2018. CA Cancer J. Clin. 2018, 68, 284-296. [CrossRef]

3. Chiang, Y.C.; Chen, C.A.; Chiang, C.J.; Hsu, T.H.; Lin, M.C.; You, S.L.; Cheng, W.F.; Lai, M.S. Trends in incidence and survival outcome of epithelial ovarian cancer: 30-year national population-based registry in Taiwan. J. Gynecol. Oncol. 2013, $24,342-351$. [CrossRef]

4. Jayson, G.C.; Kohn, E.C.; Kitchener, H.C.; Ledermann, J.A. Ovarian cancer. Lancet 2014, 384, 1376-1388. [CrossRef]

5. Coleman, R.L.; Monk, B.J.; Sood, A.K.; Herzog, T.J. Latest research and treatment of advanced-stage epithelial ovarian cancer. Nat. Rev. Clin. Oncol. 2013, 10, 211-224. [CrossRef] [PubMed]

6. Gurung, A.; Hung, T.; Morin, J.; Gilks, C.B. Molecular abnormalities in ovarian carcinoma: Clinical, morphological and therapeutic correlates. Histopathology 2013, 62, 59-70. [CrossRef] [PubMed]

7. Colombo, P.E.; Fabbro, M.; Theillet, C.; Bibeau, F.; Rouanet, P.; Ray-Coquard, I. Sensitivity and resistance to treatment in the primary management of epithelial ovarian cancer. Crit. Rev. Oncol./Hematol. 2014, 89, 207-216. [CrossRef]

8. Perren, T.J.; Swart, A.M.; Pfisterer, J.; Ledermann, J.A.; Pujade-Lauraine, E.; Kristensen, G.; Carey, M.S.; Beale, P.; Cervantes, A.; Kurzeder, C.; et al. A phase 3 trial of bevacizumab in ovarian cancer. N. Engl. J. Med. 2011, 365, 2484-2496. [CrossRef]

9. Oza, A.M.; Cook, A.D.; Pfisterer, J.; Embleton, A.; Ledermann, J.A.; Pujade-Lauraine, E.; Kristensen, G.; Carey, M.S.; Beale, P.; Cervantes, A.; et al. Standard chemotherapy with or without bevacizumab for women with newly diagnosed ovarian cancer (ICON7): Overall survival results of a phase 3 randomised trial. Lancet Oncol. 2015, 16, 928-936. [CrossRef]

10. Burger, R.A.; Brady, M.F.; Bookman, M.A.; Fleming, G.F.; Monk, B.J.; Huang, H.; Mannel, R.S.; Homesley, H.D.; Fowler, J.; Greer, B.E.; et al. Incorporation of bevacizumab in the primary treatment of ovarian cancer. N. Engl. J. Med. 2011, 365, $2473-2483$. [CrossRef]

11. González-Martín, A.; Pothuri, B.; Vergote, I.; DePont Christensen, R.; Graybill, W.; Mirza, M.R.; McCormick, C.; Lorusso, D.; Hoskins, P.; Freyer, G.; et al. Niraparib in Patients with Newly Diagnosed Advanced Ovarian Cancer. N. Engl. J. Med. 2019, 381, 2391-2402. [CrossRef] [PubMed]

12. Moore, K.; Colombo, N.; Scambia, G.; Kim, B.G.; Oaknin, A.; Friedlander, M.; Lisyanskaya, A.; Floquet, A.; Leary, A.; Sonke, G.S.; et al. Maintenance Olaparib in Patients with Newly Diagnosed Advanced Ovarian Cancer. N. Engl. J. Med. 2018, 379, 2495-2505. [CrossRef] [PubMed]

13. Mirza, M.R.; Monk, B.J.; Herrstedt, J.; Oza, A.M.; Mahner, S.; Redondo, A.; Fabbro, M.; Ledermann, J.A.; Lorusso, D.; Vergote, I.; et al. Niraparib Maintenance Therapy in Platinum-Sensitive, Recurrent Ovarian Cancer. N. Engl. J. Med. 2016, 375, $2154-2164$. [CrossRef] [PubMed]

14. Coleman, R.L.; Oza, A.M.; Lorusso, D.; Aghajanian, C.; Oaknin, A.; Dean, A.; Colombo, N.; Weberpals, J.I.; Clamp, A.; Scambia, G.; et al. Rucaparib maintenance treatment for recurrent ovarian carcinoma after response to platinum therapy (ARIEL3): A randomised, double-blind, placebo-controlled, phase 3 trial. Lancet 2017, 390, 1949-1961. [CrossRef]

15. Pujade-Lauraine, E.; Ledermann, J.A.; Selle, F.; Gebski, V.; Penson, R.T.; Oza, A.M.; Korach, J.; Huzarski, T.; Poveda, A.; Pignata, S.; et al. Olaparib tablets as maintenance therapy in patients with platinum-sensitive, relapsed ovarian cancer and a BRCA1/2 mutation (SOLO2/ENGOT-Ov21): A double-blind, randomised, placebo-controlled, phase 3 trial. Lancet Oncol. 2017, 18, 1274-1284. [CrossRef]

16. Brown, J.S.; O'Carrigan, B.; Jackson, S.P.; Yap, T.A. Targeting DNA Repair in Cancer: Beyond PARP Inhibitors. Cancer Discov. 2017, 7, 20-37. [CrossRef]

17. Pearl, L.H.; Schierz, A.C.; Ward, S.E.; Al-Lazikani, B.; Pearl, F.M. Therapeutic opportunities within the DNA damage response. Nat. Rev. Cancer 2015, 15, 166-180. [CrossRef]

18. Hanahan, D.; Weinberg, R.A. Hallmarks of cancer: The next generation. Cell 2011, 144, 646-674. [CrossRef]

19. Vilenchik, M.M.; Knudson, A.G. Endogenous DNA double-strand breaks: Production, fidelity of repair, and induction of cancer. Proc. Natl. Acad. Sci. USA 2003, 100, 12871-12876. [CrossRef]

20. Miller, R.E.; Leary, A.; Scott, C.L.; Serra, V.; Lord, C.J.; Bowtell, D.; Chang, D.K.; Garsed, D.W.; Jonkers, J.; Ledermann, J.A.; et al. ESMO recommendations on predictive biomarker testing for homologous recombination deficiency and PARP inhibitor benefit in ovarian cancer. Ann. Oncol. 2020, 31, 1606-1622. [CrossRef] 
21. Prat, J. Staging classification for cancer of the ovary, fallopian tube, and peritoneum. Int. J. Gynaecol. Obstet. 2014, 124, 1-5. [CrossRef] [PubMed]

22. Lin, P.H.; Kuo, W.H.; Huang, A.C.; Lu, Y.S.; Lin, C.H.; Kuo, S.H.; Wang, M.Y.; Liu, C.Y.; Cheng, F.T.; Yeh, M.H.; et al. Multiple gene sequencing for risk assessment in patients with early-onset or familial breast cancer. Oncotarget 2016. [CrossRef] [PubMed]

23. Li, H.; Durbin, R. Fast and accurate short read alignment with Burrows-Wheeler transform. Bioinformatics 2009, 25, 1754-1760. [CrossRef]

24. Li, H.; Handsaker, B.; Wysoker, A.; Fennell, T.; Ruan, J.; Homer, N.; Marth, G.; Abecasis, G.; Durbin, R. The Sequence Alignment/Map format and SAMtools. Bioinformatics 2009, 25, 2078-2079. [CrossRef]

25. Mimori, T.; Nariai, N.; Kojima, K.; Takahashi, M.; Ono, A.; Sato, Y.; Yamaguchi-Kabata, Y.; Nagasaki, M. iSVP: An integrated structural variant calling pipeline from high-throughput sequencing data. BMC Syst. Biol. 2013, 7 (Suppl. S6), S8. [CrossRef] [PubMed]

26. McKenna, A.; Hanna, M.; Banks, E.; Sivachenko, A.; Cibulskis, K.; Kernytsky, A.; Garimella, K.; Altshuler, D.; Gabriel, S.; Daly, M.; et al. The Genome Analysis Toolkit: A MapReduce framework for analyzing next-generation DNA sequencing data. Genome Res. 2010, 20, 1297-1303. [CrossRef]

27. Wang, K.; Li, M.; Hakonarson, H. ANNOVAR: Functional annotation of genetic variants from high-throughput sequencing data. Nucleic Acids Res. 2010, 38, e164. [CrossRef]

28. Plon, S.E.; Eccles, D.M.; Easton, D.; Foulkes, W.D.; Genuardi, M.; Greenblatt, M.S.; Hogervorst, F.B.; Hoogerbrugge, N.; Spurdle, A.B.; Tavtigian, S.V. Sequence variant classification and reporting: Recommendations for improving the interpretation of cancer susceptibility genetic test results. Hum. Mutat. 2008, 29, 1282-1291. [CrossRef]

29. Adzhubei, I.A.; Schmidt, S.; Peshkin, L.; Ramensky, V.E.; Gerasimova, A.; Bork, P.; Kondrashov, A.S.; Sunyaev, S.R. A method and server for predicting damaging missense mutations. Nat. Methods 2010, 7, 248-249. [CrossRef]

30. Kumar, P.; Henikoff, S.; Ng, P.C. Predicting the effects of coding non-synonymous variants on protein function using the SIFT algorithm. Nat. Protoc. 2009, 4, 1073-1081. [CrossRef]

31. Mathe, E.; Olivier, M.; Kato, S.; Ishioka, C.; Hainaut, P.; Tavtigian, S.V. Computational approaches for predicting the biological effect of p53 missense mutations: A comparison of three sequence analysis based methods. Nucleic Acids Res. 2006, 34, 1317-1325. [CrossRef] [PubMed]

32. Daly, M.B.; Pal, T.; Berry, M.P.; Buys, S.S.; Dickson, P.; Domchek, S.M.; Elkhanany, A.; Friedman, S.; Goggins, M.; Hutton, M.L.; et al. Genetic/Familial High-Risk Assessment: Breast, Ovarian, and Pancreatic, Version 2.2021, NCCN Clinical Practice Guidelines in Oncology. J. Natl. Compr. Canc. Netw. 2021, 19, 77-102. [CrossRef] [PubMed]

33. Hennessy, B.T.; Timms, K.M.; Carey, M.S.; Gutin, A.; Meyer, L.A.; Flake, D.D., 2nd; Abkevich, V.; Potter, J.; Pruss, D.; Glenn, P.; et al. Somatic mutations in BRCA1 and BRCA2 could expand the number of patients that benefit from poly (ADP ribose) polymerase inhibitors in ovarian cancer. J. Clin. Oncol. 2010, 28, 3570-3576. [CrossRef] [PubMed]

34. Cancer Genome Atlas Research Network. Integrated genomic analyses of ovarian carcinoma. Nature 2011, 474, 609-615. [CrossRef] [PubMed]

35. Pennington, K.P.; Walsh, T.; Harrell, M.I.; Lee, M.K.; Pennil, C.C.; Rendi, M.H.; Thornton, A.; Norquist, B.M.; Casadei, S.; Nord, A.S.; et al. Germline and somatic mutations in homologous recombination genes predict platinum response and survival in ovarian, fallopian tube, and peritoneal carcinomas. Clin. Cancer Res. 2014, 20, 764-775. [CrossRef]

36. Walsh, T.; Casadei, S.; Lee, M.K.; Pennil, C.C.; Nord, A.S.; Thornton, A.M.; Roeb, W.; Agnew, K.J.; Stray, S.M.; Wickramanayake, A.; et al. Mutations in 12 genes for inherited ovarian, fallopian tube, and peritoneal carcinoma identified by massively parallel sequencing. Proc. Natl. Acad. Sci. USA 2011, 108, 18032-18037. [CrossRef]

37. Alsop, K.; Fereday, S.; Meldrum, C.; deFazio, A.; Emmanuel, C.; George, J.; Dobrovic, A.; Birrer, M.J.; Webb, P.M.; Stewart, C.; et al BRCA mutation frequency and patterns of treatment response in BRCA mutation-positive women with ovarian cancer: A report from the Australian Ovarian Cancer Study Group. J. Clin. Oncol. 2012, 30, 2654-2663. [CrossRef]

38. Leskela, S.; Romero, I.; Cristobal, E.; Pérez-Mies, B.; Rosa-Rosa, J.M.; Gutierrez-Pecharroman, A.; Caniego-Casas, T.; Santón, A.; Ojeda, B.; López-Reig, R.; et al. Mismatch Repair Deficiency in Ovarian Carcinoma: Frequency, Causes, and Consequences. Am. J. Surg. Pathol. 2020, 44, 649-656. [CrossRef]

39. Tan, D.S.; Miller, R.E.; Kaye, S.B. New perspectives on molecular targeted therapy in ovarian clear cell carcinoma. Br. J. Cancer 2013, 108, 1553-1559. [CrossRef]

40. Jones, S.; Wang, T.L.; Shih Ie, M.; Mao, T.L.; Nakayama, K.; Roden, R.; Glas, R.; Slamon, D.; Diaz, L.A., Jr.; Vogelstein, B.; et al Frequent mutations of chromatin remodeling gene ARID1A in ovarian clear cell carcinoma. Science 2010, 330, 228-231. [CrossRef]

41. Yang, Q.; Zhang, C.; Ren, Y.; Yi, H.; Luo, T.; Xing, F.; Bai, X.; Cui, L.; Zhu, L.; Ouyang, J.; et al. Genomic characterization of Chinese ovarian clear cell carcinoma identifies driver genes by whole exome sequencing. Neoplasia 2020, 22, 399-430. [CrossRef]

42. Kim, S.I.; Lee, J.W.; Lee, M.; Kim, H.S.; Chung, H.H.; Kim, J.W.; Park, N.H.; Song, Y.S.; Seo, J.S. Genomic landscape of ovarian clear cell carcinoma via whole exome sequencing. Gynecol. Oncol. 2018, 148, 375-382. [CrossRef]

43. Murakami, R.; Matsumura, N.; Brown, J.B.; Higasa, K.; Tsutsumi, T.; Kamada, M.; Abou-Taleb, H.; Hosoe, Y.; Kitamura, S.; Yamaguchi, K.; et al. Exome Sequencing Landscape Analysis in Ovarian Clear Cell Carcinoma Shed Light on Key Chromosomal Regions and Mutation Gene Networks. Am. J. Pathol. 2017, 187, 2246-2258. [CrossRef]

44. Gounaris, I.; Brenton, J.D. Molecular pathogenesis of ovarian clear cell carcinoma. Future Oncol. (Lond. Engl.) 2015, 11, 1389-1405. [CrossRef] [PubMed] 
45. Takenaka, M.; Köbel, M.; Garsed, D.W.; Fereday, S.; Pandey, A.; Etemadmoghadam, D.; Hendley, J.; Kawabata, A.; Noguchi, D.; Yanaihara, N.; et al. Survival Following Chemotherapy in Ovarian Clear Cell Carcinoma Is Not Associated with Pathological Misclassification of Tumor Histotype. Clin. Cancer Res. 2019, 25, 3962-3973. [CrossRef] [PubMed]

46. Su, Y.F.; Tsai, E.M.; Chen, C.C.; Wu, C.C.; Er, T.K. Targeted sequencing of a specific gene panel detects a high frequency of ARID1A and PIK3CA mutations in ovarian clear cell carcinoma. Clin. Chim. Acta Int. J. Clin. Chem. 2019, 494, 1-7. [CrossRef] [PubMed]

47. Shibuya, Y.; Tokunaga, H.; Saito, S.; Shimokawa, K.; Katsuoka, F.; Bin, L.; Kojima, K.; Nagasaki, M.; Yamamoto, M.; Yaegashi, $\mathrm{N}$; ; et al. Identification of somatic genetic alterations in ovarian clear cell carcinoma with next generation sequencing. Genes Chromosomes Cancer 2018, 57, 51-60. [CrossRef] [PubMed]

48. Maru, Y.; Tanaka, N.; Ohira, M.; Itami, M.; Hippo, Y.; Nagase, H. Identification of novel mutations in Japanese ovarian clear cell carcinoma patients using optimized targeted NGS for clinical diagnosis. Gynecol. Oncol. 2017, 144, 377-383. [CrossRef]

49. Friedlander, M.L.; Russell, K.; Millis, S.; Gatalica, Z.; Bender, R.; Voss, A. Molecular Profiling of Clear Cell Ovarian Cancers: Identifying Potential Treatment Targets for Clinical Trials. Int. J. Gynecol. Cancer 2016, 26, 648-654. [CrossRef]

50. Coleman, R.L.; Fleming, G.F.; Brady, M.F.; Swisher, E.M.; Steffensen, K.D.; Friedlander, M.; Okamoto, A.; Moore, K.N.; Efrat Ben-Baruch, N.; Werner, T.L.; et al. Veliparib with First-Line Chemotherapy and as Maintenance Therapy in Ovarian Cancer. $N$ Engl. J. Med. 2019, 381, 2403-2415. [CrossRef]

51. Ray-Coquard, I.; Pautier, P.; Pignata, S.; Pérol, D.; González-Martín, A.; Berger, R.; Fujiwara, K.; Vergote, I.; Colombo, N.; Mäenpää, J.; et al. Olaparib plus Bevacizumab as First-Line Maintenance in Ovarian Cancer. N. Engl. J. Med. 2019, 381, 2416-2428. [CrossRef] [PubMed]

52. Konstantinopoulos, P.A.; Norquist, B.; Lacchetti, C.; Armstrong, D.; Grisham, R.N.; Goodfellow, P.J.; Kohn, E.C.; Levine, D.A.; Liu, J.F.; Lu, K.H.; et al. Germline and Somatic Tumor Testing in Epithelial Ovarian Cancer: ASCO Guideline. J. Clin. Oncol. 2020, 38, 1222-1245. [CrossRef]

53. Vergote, I.; Banerjee, S.; Gerdes, A.M.; van Asperen, C.; Marth, C.; Vaz, F.; Ray-Coquard, I.; Stoppa-Lyonnet, D.; Gonzalez-Martin, A.; Sehouli, J.; et al. Current perspectives on recommendations for BRCA genetic testing in ovarian cancer patients. Eur. J. Cancer 2016, 69, 127-134. [CrossRef]

54. Lancaster, J.M.; Powell, C.B.; Chen, L.M.; Richardson, D.L. Society of Gynecologic Oncology statement on risk assessment for inherited gynecologic cancer predispositions. Gynecol. Oncol. 2015, 136, 3-7. [CrossRef]

55. Norquist, B.M.; Brady, M.F.; Harrell, M.I.; Walsh, T.; Lee, M.K.; Gulsuner, S.; Bernards, S.S.; Casadei, S.; Burger, R.A.; Tewari, K.S.; et al. Mutations in Homologous Recombination Genes and Outcomes in Ovarian Carcinoma Patients in GOG 218: An NRG Oncology/Gynecologic Oncology Group Study. Clin. Cancer Res. 2018, 24, 777-783. [CrossRef]

56. Loveday, C.; Turnbull, C.; Ramsay, E.; Hughes, D.; Ruark, E.; Frankum, J.R.; Bowden, G.; Kalmyrzaev, B.; Warren-Perry, M.; Snape, K.; et al. Germline mutations in RAD51D confer susceptibility to ovarian cancer. Nat. Genet. 2011, 43, 879-882. [CrossRef]

57. McCabe, N.; Turner, N.C.; Lord, C.J.; Kluzek, K.; Bialkowska, A.; Swift, S.; Giavara, S.; O'Connor, M.J.; Tutt, A.N.; Zdzienicka, M.Z.; et al. Deficiency in the repair of DNA damage by homologous recombination and sensitivity to poly(ADP-ribose) polymerase inhibition. Cancer Res. 2006, 66, 8109-8115. [CrossRef]

58. Hodgson, D.R.; Dougherty, B.A.; Lai, Z.; Fielding, A.; Grinsted, L.; Spencer, S.; O'Connor, M.J.; Ho, T.W.; Robertson, J.D.; Lanchbury, J.S.; et al. Candidate biomarkers of PARP inhibitor sensitivity in ovarian cancer beyond the BRCA genes. Br. J. Cancer 2018, 119, 1401-1409. [CrossRef]

59. Swisher, E.M.; Lin, K.K.; Oza, A.M.; Scott, C.L.; Giordano, H.; Sun, J.; Konecny, G.E.; Coleman, R.L.; Tinker, A.V.; O’Malley, D.M.; et al. Rucaparib in relapsed, platinum-sensitive high-grade ovarian carcinoma (ARIEL2 Part 1): An international, multicentre, open-label, phase 2 trial. Lancet Oncol. 2017, 18, 75-87. [CrossRef]

60. Bonnet, C.; Krieger, S.; Vezain, M.; Rousselin, A.; Tournier, I.; Martins, A.; Berthet, P.; Chevrier, A.; Dugast, C.; Layet, V.; et al. Screening BRCA1 and BRCA2 unclassified variants for splicing mutations using reverse transcription PCR on patient RNA and an ex vivo assay based on a splicing reporter minigene. J. Med. Genet. 2008, 45, 438-446. [CrossRef]

61. Cartegni, L.; Chew, S.L.; Krainer, A.R. Listening to silence and understanding nonsense: Exonic mutations that affect splicing. Nat. Rev. Genet. 2002, 3, 285-298. [CrossRef]

62. Li, H.; LaDuca, H.; Pesaran, T.; Chao, E.C.; Dolinsky, J.S.; Parsons, M.; Spurdle, A.B.; Polley, E.C.; Shimelis, H.; Hart, S.N.; et al. Classification of variants of uncertain significance in BRCA1 and BRCA2 using personal and family history of cancer from individuals in a large hereditary cancer multigene panel testing cohort. Genet. Med. 2020, 22, 701-708. [CrossRef]

63. Alexandrov, L.B.; Kim, J.; Haradhvala, N.J.; Huang, M.N.; Tian Ng, A.W.; Wu, Y.; Boot, A.; Covington, K.R.; Gordenin, D.A.; Bergstrom, E.N.; et al. The repertoire of mutational signatures in human cancer. Nature 2020, 578, 94-101. [CrossRef]

64. Toland, A.E.; Forman, A.; Couch, F.J.; Culver, J.O.; Eccles, D.M.; Foulkes, W.D.; Hogervorst, F.B.L.; Houdayer, C.; Levy-Lahad, E.; Monteiro, A.N.; et al. Clinical testing of BRCA1 and BRCA2: A worldwide snapshot of technological practices. NPJ Genom. Med. 2018, 3, 7. [CrossRef] 